\title{
TAXONOMIC SURVEY OF BENTHIC DIATOMS IN NEUM BAY, SOUTHEASTERN ADRIATIC
}

\author{
Dubravka Hafner ${ }^{1}$, Nenad Jasprica ${ }^{2 *}$ \& Ana Car ${ }^{2}$ \\ ${ }^{1}$ Bartulovići 4, HR-20357 Blace, Croatia \\ ${ }^{2}$ Laboratory of Plankton Ecology and Population Genetics, Institute for Marine and Coastal \\ Research, University of Dubrovnik, Kneza Damjana Jude 12, PO Box 83, \\ HR-20000 Dubrovnik, Croatia
}

\begin{abstract}
Hafner, D., Jasprica, N. \& Car, A.: Taxonomic survey of benthic diatoms in Neum Bay, southeastern Adriatic. Nat. Croat., Vol. 27, No. 1, 1-26, 2018, Zagreb.

The objective of this work was to contribute to the knowledge of microphytobenthos in the Adriatic Sea, by studying for the first time the benthic diatom communities in Neum Bay in Bosnia and Herzegovina. For this purpose, the benthic diatoms naturally growing on stones and macroalgae were identified at one station in the small semi-enclosed oligotrophic Neum Bay during 2010 and 2011. A total of 24 samples were collected at two different depths, $0.5 \mathrm{~m}$ and $8 \mathrm{~m}$, and analysed with light and scanning electron microscopy. Altogether, 425 pennate and 58 centric taxa (species and infraspecific taxa) belonging to 60 families and 115 genera were noted. Genera with the greatest number of taxa were: Mastogloia (46 taxa), Navicula (36), Diploneis (35), Nitzschia (34), Amphora (31), Cocconeis (27), Achnanthes (14), Halamphora (12), Lyrella (11), and Surirella and Licmophora (10 each). Amphora bigibba var. interrupta and Cocconeis scutellum were the most frequent taxa, being present in $87.5 \%$ of the samples. In total, 142 taxa were found only once (sporadic taxa). Although benthic diatom richness is high in the bay, taxa are apparently not distributed evenly temporally. Consistent quantitative and qualitative data are still needed for a better determination of the seasonal and spatial changes of the epilithic assemblages in the region.
\end{abstract}

Keywords: Bacillariophyta, shallow oligotrophic bay, species identification, biodiversity, Bosnia and Herzegovina.

Hafner, D., Jasprica, N. \& Car, A.: Taksonomska analiza bentoskih dijatomeja u Neumskom zaljevu, jugoistočna obala Jadrana. Nat. Croat., Vol. 27, No. 1, 1-26, 2018, Zagreb.

Cilj rada bio je doprinijeti poznavanju mikrofitobentosa u Jadranskom moru istražujući po prvi put bentoske dijatomejske zajednice u Neumskom zaljevu u Bosni i Hercegovini. Bentoske dijatomeje su određene na jednoj postaji u poluzatvorenom oligotrofnom Neumskom zaljevu 2010. i 2011. Sakupljeno je 24 uzoraka na dubinama 0,5 i $8 \mathrm{~m}$, a analizirani su s pomoću svjetlosnog i skenirajućeg elektronskog mikroskopa. U uzorcima je utvrđeno 425 penatnih i 58 centričnih svojti (vrsta i nižih taksonomskih kategorija) dijatomeja unutar 60 porodica i 115 rodova. Najveći broj svojti nađen je unutar sljedećih rodova: Mastogloia (46 svojti), Navicula (36), Diploneis (35), Nitzschia (34), Amphora (31), Cocconeis (27), Achnanthes (14), Halamphora (12), Lyrella (11) te Surirella i Licmophora (svaki po 10 svojti). Najčešće svojte su Amphora bigibba var. interrupta i Cocconeis scutellum s 87,5\% učestalosti u ukupnom broju uzoraka. Utvrđene su 142 sporadične svojte (utvrđene u samo jednom uzorku). Iako je broj svojti relativno visok, nije utvrđena pravilnost u njihovoj vremenskoj raspodjeli. Radi preciznijeg utvrđivanja sezonskih i prostornih promjena strukture epilitskih dijatomejskih zajednica, potrebni su detaljniji kvalitativni i kvantitativni podaci.

Ključne riječi: Bacillariophyta, plitki oligotrofni zaljev, taksonomska istraživanja, bogatstvo svojti, Bosna i Hecegovina

\footnotetext{
*Corresponding author, E-mail: nenad.jasprica@unidu.hr
} 


\section{INTRODUCTION}

Benthic diatoms are colonial or unicellular diatoms, free living or attaching to the substratum by gelatinous extrusion. They are mostly associated with substrata throughout their life cycles. Some of them move actively in sediments and sometimes resuspend in the water column. Benthic diatoms are the most abundant and diversified part of marine microphytobenthos playing an important role in marine ecosystems (FALKоwsкi et al., 2004). These organisms are used as water quality indicators as well as in palaeoecological reconstructions due to their ecophysiological features (CIBIC \& Blasutto, 2011; Stevenson \& Pan, 1999). In addition, knowledge of the structure of the benthic diatom community and the autoecology of individual taxa is a unique source of information in the study of the dynamics of the marine microphytobenthos.

In the Mediterranean Sea, Çolak Sabanci \& Koray (2010), ÇolaK Sabanci (2012) and Álvarez-BlanCO \& BlANCO (2014) recently investigated the structure and taxonomic composition of benthic diatom communities. In the Adriatic Sea, the diatom taxa from natural sediment samples and artificial substrata of the Gulf of Trieste (BARTOLE et al., 1991-94, Sdrigotti et al., 1999; Munda, 2005), the Venice lagoon (Tolomio \& Andreoli, 1989, Tоlomio et al., 1999; Facca et al., 2002; Tolomio et al. 2002; FACCA \& SFriso, 2007), the northwestern Adriatic coast (Tотті, 2003; Tотті et al., 2007; Franzo et al., 2015, and references therein) were reported. Several studies on epibiontic diatom communities on marine hydroids in the Ligurian Sea have also been reported (RomAGNoli et al., 2007, 2014, and references therein). Finally, a checklist on microphytobenthos of the Italian seas, including benthic diatom taxa, was compiled by Ciвic \& FACCA (2010).

On the other hand, to the best of our knowledge, the structure and taxonomic composition of benthic diatoms have been only rarely taken into consideration in the eastern Adriatic Sea (Burić et al., 2004; Mino \& Witkowski, 2005; Caput et al., 2008; Levkov et al., 2010; CAR et al., 2012; NenAdović et al., 2015; MejAndžić et al., 2015). Diatoms were investigated synoptically and mostly related to estuaries or coastal wetlands.

The objective of this work was to contribute to the knowledge of microphytobenthos in the Adriatic Sea, by studying for the first time the benthic diatom communities in Neum Bay in Bosnia and Herzegovina.

\section{MATERIAL AND METHODS}

\section{Study area}

Bosnia and Herzegovina has a total sea area of ca. $8 \mathrm{~km}^{2}$ with only 21.2 kilometers of coastline (Fig. 1). The coast is low and rocky, built up of karstified carbonates and represents only $0.26 \%$ of the total length of the Adriatic coastline (BLAKE \& Topalović, 1996; PikelJ \& JuRAčić, 2013). In fact, the sea area of the country is represented by the small, semi-enclosed Neum Bay, located between mainland and the $7 \mathrm{~km}$-long Klek Peninsula (Dupčıć-RAdić et al., 2013).

Neum Bay is part of the larger Mali Ston Bay, which is enclosed on the seaward side by the $62 \mathrm{~km}$ long Pelješac peninsula. It expands to the northwest and connects with the Neretva River channel which is linked with the open sea. Due to its ecological and economic importance, this area with a centuries-long mariculture tradition was in 1983 proclaimed the Mali Ston Bay and Malo More Special Marine Reserve, including Neum Bay. The most important factors that affect the ecological conditions in Mali Ston Bay, 
primarily salinity and nutrient enrichment, are the inflow of freshwater from the Neretva River in the outer part of the bay and from underwater springs ("vruljas") in the inner part (JASPRICA, 1989; JASPRICA et al., 1997; JASPRICA \& CARIĆ, 2001; ČAlić et al., 2013).

The average and maximum water depth in Neum Bay are 17 and $27 \mathrm{~m}$, respectively. The bottom structure ranges from sandy sediments in the central part of the bay to rocks where the system communicates with the coastline. The town of Neum (population of 3,236 estimated 2013) is the only coastal settlement in Bosnia and Herzegovina and an important tourism destination for the country, which means that the area is under significant anthropogenic pressure during summer.

Additional details on the area are given by JASPRICA et al. (2012) and ČAlić et al. (2013).

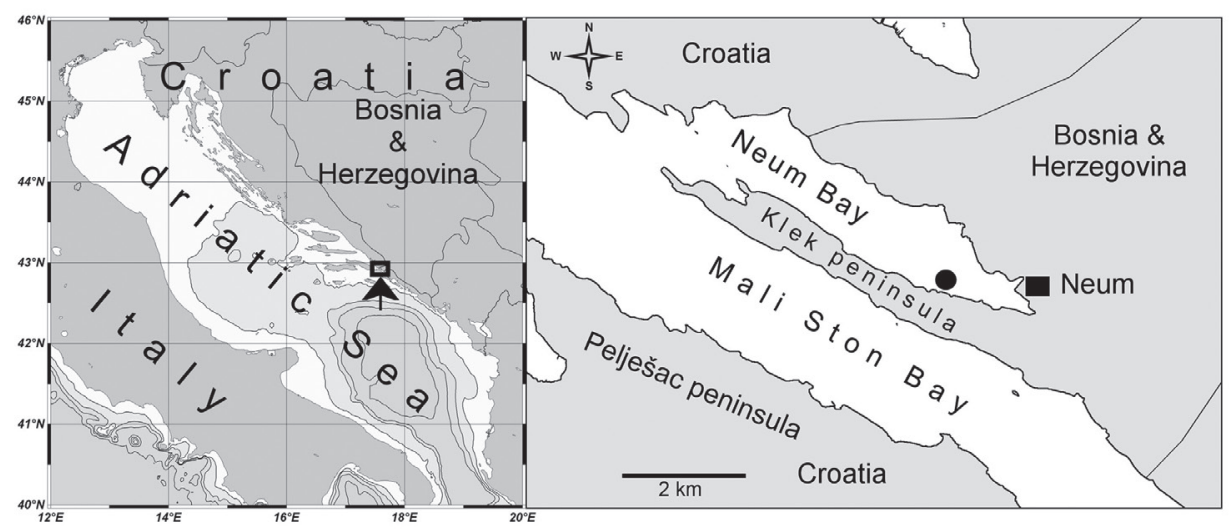

Fig. 1. Sampling location (•) in Neum Bay, Bosnia and Herzegovina.

\section{Sampling and analyses}

The investigated station (WGS84 42 $54^{\prime} 58.7^{\prime \prime} \mathrm{N} ; 1^{\circ} 36^{\prime} 17.6^{\prime \prime} \mathrm{E}$ ) was located in the southern part of Neum Bay (Fig. 1). Samples were collected from a depth of $0.5 \mathrm{~m}$ in March, May and July of 2010 and at monthly intervals from January to December 2011 (see Tab. 1 for sampling design). In addition, samples were also collected from a depth of $8 \mathrm{~m}$ from April to December 2011. Altogether, 24 samples were analysed.

Temperature was measured with an inverted thermometer. Water samples for salinity were taken by 5-L Niskin bottles and determined by argentometric titration (GRAssHOFF et al., 1983).

Tab. 1. Sampling design of benthic diatom survey conducted in the Neum Bay, Bosnia and Herzegovina $(\bullet$ - sampled; $\otimes$ - not sampled) in 2010 and 2011.

\begin{tabular}{|l|c|c|c|c|c|c|c|c|c|c|c|c|c|c|c|}
\hline Years & \multicolumn{9}{|c|}{2010} & \multicolumn{10}{|c|}{2011} \\
\hline Months & 3 & 5 & 7 & 1 & 2 & 3 & 4 & 5 & 6 & 7 & 8 & 9 & 10 & 11 & 12 \\
\hline $0.5 \mathrm{~m}$ & $\bullet$ & $\bullet$ & $\bullet$ & $\bullet$ & $\bullet$ & $\bullet$ & $\bullet$ & $\bullet$ & $\bullet$ & $\bullet$ & $\bullet$ & $\bullet$ & $\bullet$ & $\bullet$ & $\bullet$ \\
\hline $8.0 \mathrm{~m}$ & $\otimes$ & $\otimes$ & $\otimes$ & $\otimes$ & $\otimes$ & $\otimes$ & $\bullet$ & $\bullet$ & $\bullet$ & $\bullet$ & $\bullet$ & $\bullet$ & $\bullet$ & $\bullet$ & $\bullet$ \\
\hline
\end{tabular}


The sampling points were located at the distance of $1 \mathrm{~m}$ for the shallow samples and $25 \mathrm{~m}$ for the deeper samples from the coastline due to the small bathymetric gradients. The diatom samples were obtained by scraping of the submersed stones and collecting attached macroalgae [mostly Cystoseira spp., Codium vermilara (Olivi) Delle Chiaje, Ceramium spp., Dictyota dichotoma (Hudson) J.V.Lamouroux]. To identify epilithic diatoms, stones of $15-20 \mathrm{~cm}$ in diameter were collected. Deeper stones were collected by scuba diving. Stones were collected as randomly as possible amongst those that are not completely covered by filamentous algae and on which the diatom biofilm was visible. Stones were put into a plastic bag of $1 \mathrm{~L}$ in which $200 \mathrm{~mL}$ of distilled water was added. The upper parts of the stones were rubbed with a toothbrush and the mixture was decanted into $250 \mathrm{~mL}$ polyethylene bottles (Winter \& Duthie, 2000). The samples were preserved with a final concentration of $4 \%$ formaldehyde.

In the laboratory, the material collected was cleaned of organic material for light (LM) and scanning electron microscopy (SEM) observations. Material was treated with sulfuric acid-potassium permanganate, decolorized with oxalic acid, and acid residues were removed by repeated decantation. The cleaned material was pipetted onto ethanol-cleaned cover-slips and left to air-dry, before mounting in Canada balsam when they were ready for light microscopic examination as permanent slides (HASLE \& FRYXELL, 1970). In order to register all diatom taxa, the entire surface area of each permanent slide has been surveyed. Whenever possible, diatoms were identified to species rank and counted at 1000 x magnification by phase-contrast optics with a Microstar binocular microscope (AO Scientific Instruments), 100 x PlanApo oil immersion objective. Slides have been deposited in the diatom collection of the Institute for Marine and Coastal Research, University of Dubrovnik, Dubrovnik, Croatia.

Ultrastructural analysis was performed using SEM. A drop of the cleaned sample was air-dried overnight on aluminium stubs and coated with gold-palladium or osmium. SEM observations were made at the Warsaw University of Technology, Faculty of Materials Science and Engineering, using a Hitachi S-3500, SU-70 and SEM/ STEM S-5500.

Identifications were made following Peragallo \& Peragallo (1897-1908), Hendey (1964), Ricard (1974, 1975, 1977), Poulin et al. (1984, 1990), Bérard-Therriault et al. (1986, 1987), Hartley (1986), Snoeijs (1993, 1999), Snoeijs \& Potapova (1995), Snoeijs \& Kasperoviciené (1996), Snoeijs \& Balashlova (1998), Hartley et al. (1996), and WitKowsкi et al. (2000). Nomenclature and data on general environments of taxa follow AlgaeBase (Guiry \& Guiry, 2017), and only partially Álvarez-Blanco \& Blanco (2014).

\section{RESULTS}

\section{Environmental conditions}

In 2011, surface water temperature varied between 10.0 and $27.1^{\circ} \mathrm{C}$, and maximum was recorded in September, while the minimum was noted in January (Tab. 2). The salinity ranged between 32.0 in April and 38.4 in December. Warm summer months were characterized by lower salinity, while in the cooler months of winter and spring salinity was mostly related to the precipitation regime and the extremely dynamic water flow from the underwater karstic springs ("vruljas") that feed the bay. 
Tab. 2. Monthly distribution of the temperature and salinity on the surface ( $0.5 \mathrm{~m}$ depth) in Neum Bay in 2010 and 2011.

\begin{tabular}{|l|c|c|c|c|c|c|c|c|c|c|c|c|c|c|c|}
\hline Years & \multicolumn{3}{|c|}{2010} & \multicolumn{10}{c|}{2011} \\
\hline Months & 3 & 5 & 7 & 1 & 2 & 3 & 4 & 5 & 6 & 7 & 8 & 9 & 10 & 11 & 12 \\
\hline Temperature $\left({ }^{\circ} \mathrm{C}\right)$ & 10.4 & 15.9 & 25.2 & 10.0 & 10.9 & 10.2 & 15.9 & 15.5 & 22.5 & 24.9 & 26 & 27.1 & 23.1 &. & 15.7 \\
\hline Salinity & 36.7 & 37.2 & 36.4 & 34.2 & 33.5 & 36.6 & 32.0 & 36.9 & 34.9 & 35.6 & 34.7 & 35.2 &. &. & 38.4 \\
\hline
\end{tabular}

\section{Taxonomic composition of the benthic diatom community}

During this study, a total of 483 specific and infraspecific diatom taxa were identified in Neum Bay (Tab. 3). The list of taxa included 425 pennate and 58 centric diatoms belonging to all three recognized diatom classes: Coscinodiscophyceae, Mediophyceae and Bacillariophyceae (Medlin \& Kaczmarska, 2004), and 60 families (Tab. 3). Among them, two taxa were included in Bacillariophyta incertae sedis (sensu Guiry \& Guiry, 2017). Ten families were included in Coscinodiscophyceae, 11 in Mediophyceae and 39 in Bacillariophyceae.

Families with the greatest number of genera were: Naviculaceae (8 genera), Bacillariaceae (6), Achnanthidiaceae (6), Surirellaceae (6), Fragilariaceae (6), Eupodiscaceae (6) and Plagiogrammaceae (4). Additionally, the highest number of species and infraspecific taxa belonged to Naviculaceae (56).

Altogether, 115 genera were found in all samples. Thirteen genera were included in Coscinodiscophyceae, 17 in Mediophyceae and 87 in Bacillariophyceae. Genera with the greatest number of taxa were: Mastogloia (46 taxa), Navicula (36), Diploneis (35), Nitzschia (34), Amphora (31), Cocconeis (27), Achnanthes (14), Halamphora (12), Lyrella (11) and Surirella and Licmophora (10 each). In total, 24 genera had two taxa each, while 60 were composed of one taxon only. Regarding the habitat type (sensu GuIRY \& GuIRY, 2017), the greatest number of diatom taxa (152) have been characterized as exclusively marine, 15 are brackish and 29 exclusively freshwater diatoms, while one (Mastogloia exigua) has been characterized as exclusively a soil diatom. Among truly marine diatoms, three Amphora taxa (Amphora bigibba var. interrupta, A. hyalina and A. laevissima) showed high frequency of appearance and were found in more than $50 \%$ of the samples.

However, Amphora bigibba var. interrupta and Cocconeis scutellum were the most frequent taxa, being present in $87.5 \%$ of the samples. Taxa with high frequencies (79.183.3\%) were: Achnanthes brevipes (83.3\%), Toxarium undulatum (79.1\%) and Licmophora remulus (79.1\%), while others (centric diatoms Actinocyclus subtilis, Biddulphia biddulphiana; and pennates Grammatophora oceanica, Halamphora coffeiformis, Brebissonia lanceolata, Coronia decorus, Synedra fulgens, Trachyneis aspera and Surirella fastuosa, Fig. 2) were found from $62.5 \%$ to $66.7 \%$ of the samples. Altogether, 34 taxa were found in at least $50 \%$ or more of the samples and could be characterised as taxa with higher frequency of appearance. In total, 142 taxa were found only once (sporadic taxa) during the investigated period (Tab. 3).

Some taxa appeared only in particular seasons and/or depths, but the determination of the relationship between their appearance and abundances, and the ecological conditions in the bay falls outside of the scope of the present paper. 

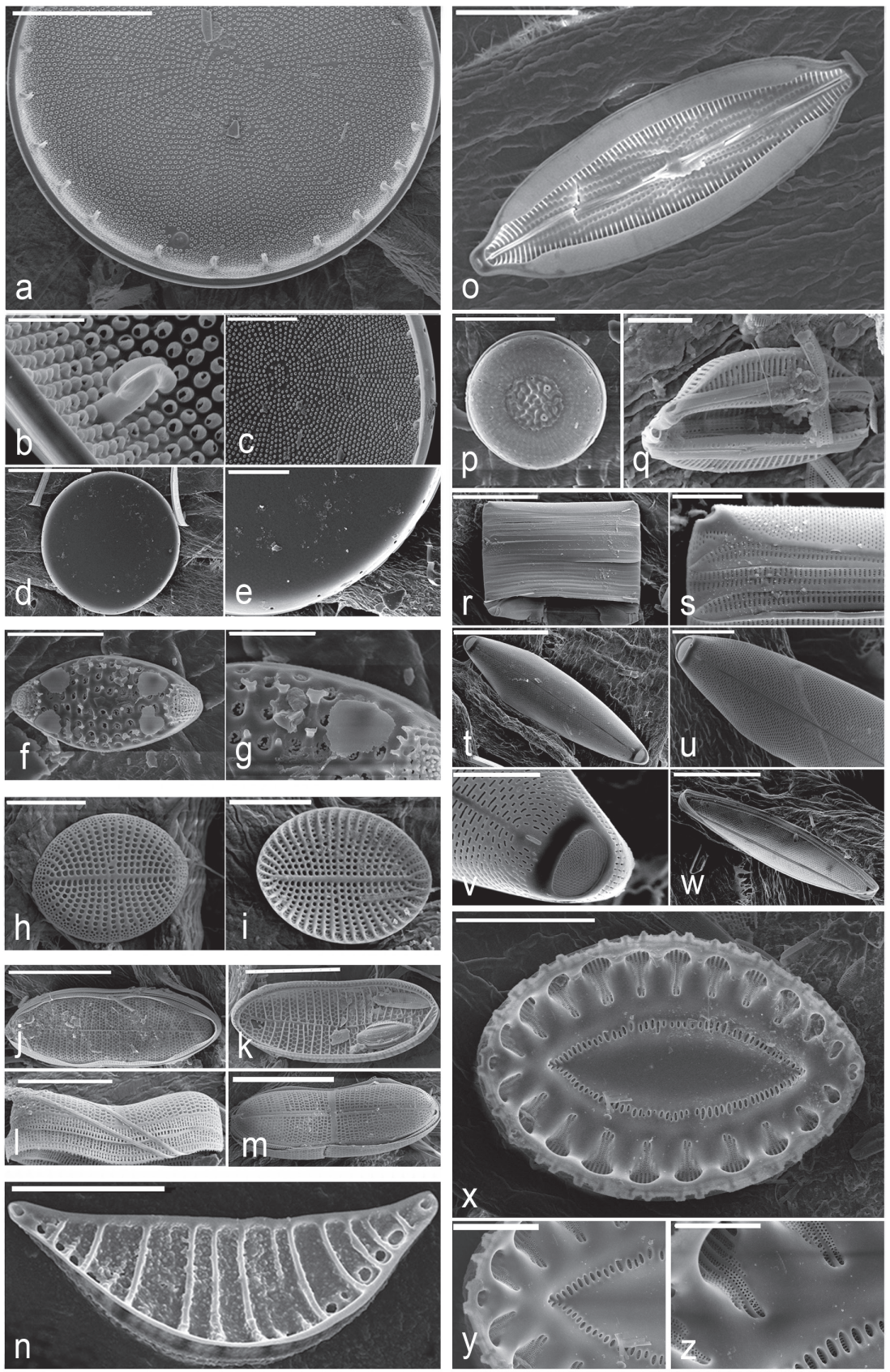

Fig. 2. Scanning electron microscope (SEM) micrographs of benthic diatoms in the Neum Bay: a-e) Actinocyclus subtilis (W.Gregory) Ralfs; f-g) Dimeregramma minus (W.Gregory) Ralfs; h-i) Cocconeis scutellum Ehrenberg; j-m) Achnanthes brevipes C.Agardh; n) Rhopalodia pacifica Krammer; o) Mastogloia ignorata Hustedt; p) Cyclotella meneghiniana Kützing; q) Halamphora coffeiformis (C.Agardh) Levkov; r-w) Striatella unipunctata (Lyngbye) C.Agardh; $\mathrm{x}-\mathrm{z}$ ) Surirella fastuosa (Ehrenberg) Ehrenberg. Scale bars $=40 \mu \mathrm{m}(\mathrm{d}), 30$ $\mu \mathrm{m}(\mathrm{j}, \mathrm{m}, \mathrm{r}, \mathrm{t}), 20 \mu \mathrm{m}(\mathrm{a}, \mathrm{k}, \mathrm{l}, \mathrm{w}, \mathrm{x}), 10 \mu \mathrm{m}(\mathrm{c}, \mathrm{e}, \mathrm{h}, \mathrm{i}, \mathrm{n}, \mathrm{o}, \mathrm{u}, \mathrm{y}), 5 \mu \mathrm{m}(\mathrm{f}, \mathrm{p}, \mathrm{q}, \mathrm{s}, \mathrm{v}, \mathrm{z}), 2 \mu \mathrm{m}(\mathrm{b}, \mathrm{g})$. 


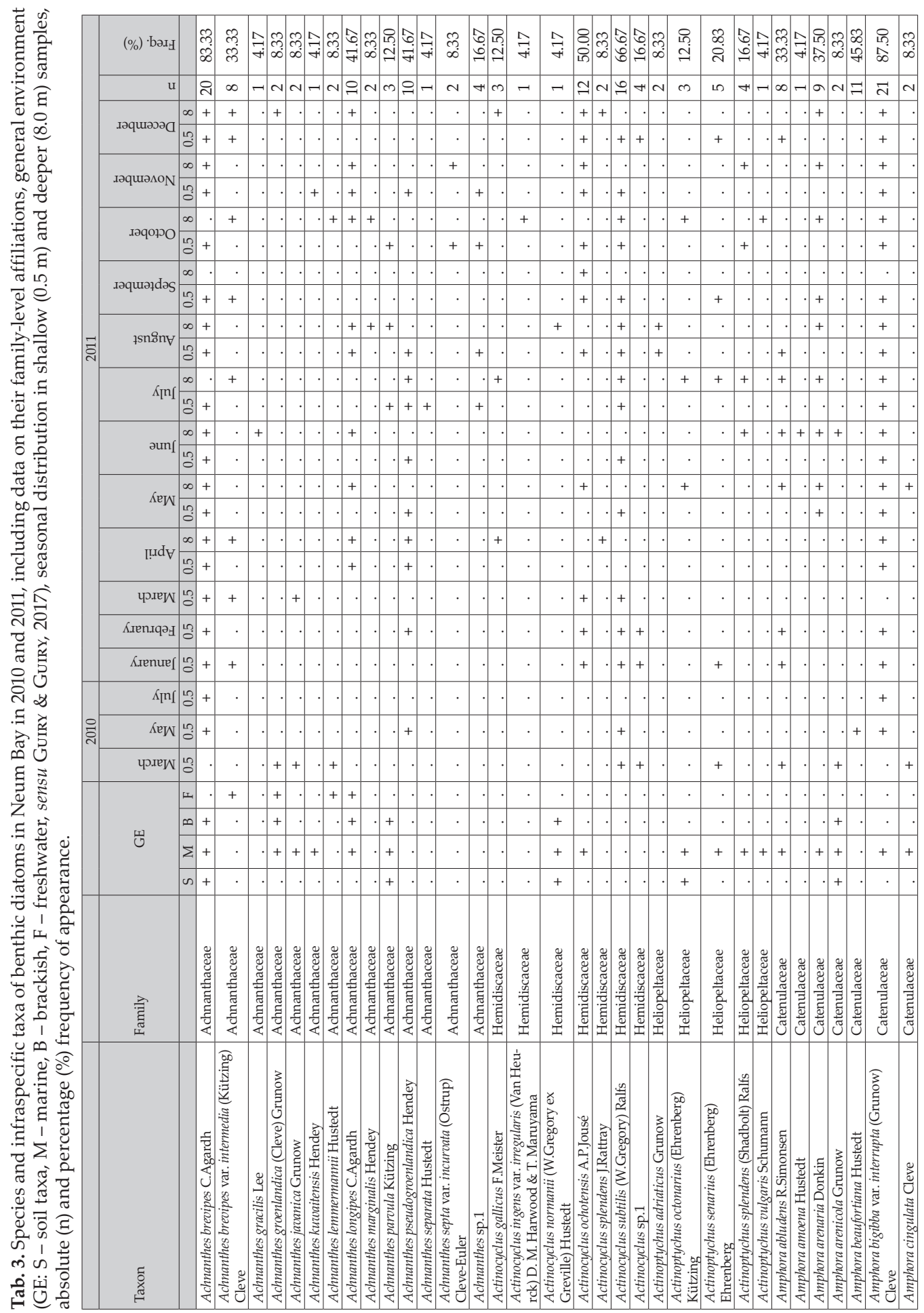




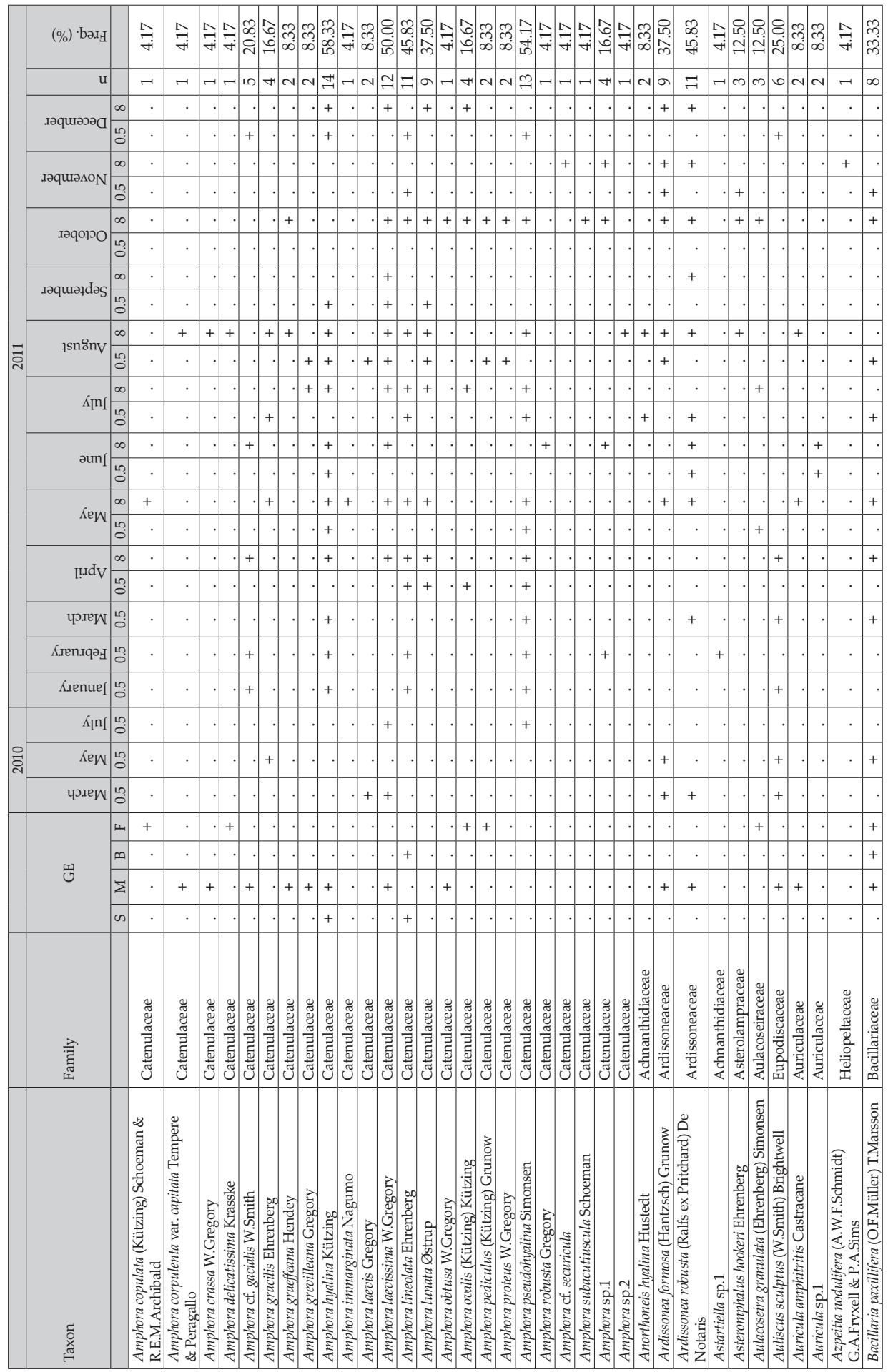




\begin{tabular}{|c|c|c|c|c|c|c|c|c|c|c|c|c|c|c|c|c|c|c|c|c|c|c|c|c|c|c|c|c|c|c|c|c|c|c|}
\hline & (\%) ·bə⿸广 & & $\begin{array}{l}\text { के } \\
\infty \\
\infty\end{array}$ & $\begin{array}{c}\infty \\
\infty \\
\infty\end{array}$ & $\begin{array}{l}\text { ณ } \\
\text { i }\end{array}$ & $\stackrel{7}{F}$ & & & $\stackrel{\vec{f}}{\vec{t}}$ & $\begin{array}{c}m \\
\infty \\
\infty\end{array}$ & مొ & $\begin{array}{c}\infty \\
\infty \\
\infty \\
\infty\end{array}$ & 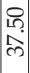 & $\overrightarrow{\vec{t}}$ & $\stackrel{\vec{\partial}}{\overrightarrow{+}}$ & $\overrightarrow{\vec{t}}$ & & $\begin{array}{l}\text { के } \\
\text { लें. }\end{array}$ & & & $\stackrel{\vec{f}}{\vec{H}}$ & $\underset{7}{7}$ & $\underset{7}{\stackrel{D}{7}}$ & & & & 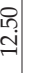 & $\begin{array}{c}\infty \\
\infty \\
\infty \\
\infty\end{array}$ & & $\begin{array}{c}\infty \\
\infty \\
\infty\end{array}$ & $\stackrel{\triangle}{\overrightarrow{+}}$ & $\stackrel{+}{A}$ & & \\
\hline & & & $\infty$ & N & $m$ & -1 & $\div$ & + & - & $N$ & $\stackrel{20}{2}$ & N & $a$ & -1 & -1 & - & in & $\infty$ & 의 & 6 & $\rightarrow$ & - & - & $\mathrm{N}$ & - & $m$ & $m$ & $N$ & a & $N$ & - & -1. & -1 & $r$ \\
\hline & & $\infty$ & & & . & & + & + & & . & . & & + & & & & & • & 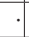 & + & . & $\cdot$ & & & & & & . & & & . & & & \\
\hline & ${ }_{0}$ & 10 & 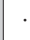 & & - & & + & & & . & + & & & & & & . & . & + & & . & $\cdot$ & & & & $\cdot$ & & . & & & . & & & \\
\hline & & $\infty$ & + & & . & & + & + & & . & . & & & & & & & & + & $\cdot$ & . & $\cdot$ & & & & . & 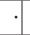 & . & & & . & & & \\
\hline & $\mathrm{O}_{\mathrm{N}}$ & 10 & • & & . & & ] & . & + & . & . & & & & & . & . & + & &. & . & . & . & & & + & & . & & . & . & & & \\
\hline & & $\infty$ & + & & . & & + & + & & . & + & & & 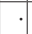 & $\cdot$ & $\cdot$ & $\cdot$ & + & + & + & . & + & & & $\cdot$ & & + & . & & + & . & & & \\
\hline & 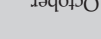 & 10 & + & & . & & + & & & $\cdot$ & + & & s & & & $\cdot$ & $\cdot$ & $\cdot$ & & & . & . & . & & & & & . & & & . & & & \\
\hline & & $\infty$ & . & & . & & + & & & . &. & & + & $\cdot$ & . & 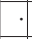 & + & $\cdot$ & . & $\cdot$ & . & $\cdot$ & $\cdot$ & & . & . & & . & & & + & & & \\
\hline & 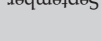 & 10 & + & & . & & + & & & + & + & & & & & & & $\cdot$ & . & . & . & $\cdot$ & . & & & + & & . & & & . & & & \\
\hline & sengnt & $\infty$ & + & & . & & + & & & . & . & & + & + & + & $\cdot$ & . & + & + & & . & $\cdot$ & + & & & & & . & & & . & + & & \\
\hline त & $v_{V}$ & $\stackrel{10}{\circ}$ & + & & . & & $\cdot$ & & & $\cdot$ & + & & & & & & $\cdot$ & . & + & & . & $\cdot$ & & & & & . & . & & & . & & & \\
\hline & & $\infty$ & + & & + & & + & & & $\cdot$ & + & & & & & & . & . & + & & . & $\cdot$ & . & & & & + & . & & & . & & & + \\
\hline & $x\left[I_{1}\right.$ & 10 & - & & . & & $\cdot$ & & & . & + & & + & & & . & . & . & + & $\cdot$ & . & . & . & & $\cdot$ & & & . & & & . & & + & \\
\hline & & $\infty$ & + & & . & & + & & & . & + & & + & 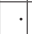 & & + & + & + & + & $\cdot$ & . & $\cdot$ & $\cdot$ & . & $\cdot$ & & & $\cdot$ & & & . & & & \\
\hline & क्या & 10 & & & . & & + & & & . & + & & + & 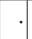 & & & + & • & & $\cdot$ & . & $\cdot$ & $\cdot$ & & $\cdot \cdot$ & & & . & & & . & & & \\
\hline & Spiot & $\infty$ & & & . & & + & & & . & + & & + & & & & + & + & & + & . & $\cdot$ & 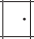 & & . & & & . & & & . & & & \\
\hline & & $\stackrel{10}{\circ}$ & . & & . & & & . & & . & + & & & & & & + & + & & + & . & $\cdot$ & . & & + & & & . & & & . & & & \\
\hline & udy & $\infty$ & + & & + & & . & + & & $\cdot$ & . & & 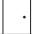 & & & & & + & & + & . & $\cdot$ & . & 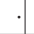 & & & + & $\cdot$ & + & & . & & & \\
\hline & {$\left[!+u \nabla_{\nabla}\right.$} & นั้ & & & $\cdot$ & & $\cdot$ & 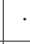 & & . & + & & & & & & & . & & + & . & $\cdot$ & & . & & & & . & & & . & & & \\
\hline & чргеW & 10 & & & . & & + & & & . & + & & & & & & &. & & & . & $\cdot$ & & + & & & & + & & & . & & & \\
\hline & К.еnıqә & 10 & & & . & & & & & . & + & + & + & & & & & 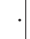 & & & . & $\cdot$ & & + & & & & . & & & . & & & \\
\hline & Krenue & 10 & & + & . & & + & & & . & + & & & & & & & & + & & . & $\cdot$ & & & & & & . & & & . & & & \\
\hline & $\left.\kappa_{[} \mathrm{n}\right]$ & $\stackrel{10}{\circ}$ & & & . & & + & & & . & . & & & & & & &. & & & . & $\cdot$ & & & & & & . & & & . & & & \\
\hline 윰 & ${ }^{\alpha} \mathrm{e} W$ & $\stackrel{10}{\circ}$ & & & . & + & + & & & - & . & & + & & & & & & + & & + & · & & & & + & & . & & + & . & & & \\
\hline & पग्रू & 10 & & + & + & & & & & + & $\cdot$ & + & & & & & & + & & & . & . & & & & & & + & & & · & & & \\
\hline & & 工 & . & & . & & & . & & . & + & & & + & 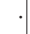 & . & . & $\cdot$ & & . & + & . & , & & & 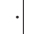 & & . & & 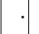 & . & & & + \\
\hline & & $\infty$ & + & & . & . & 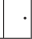 & + & & . & + & & . & & & & & + & & & + & . & . & & & & . & + & + & . & . & & & + \\
\hline & ज & $\Sigma$ & + & & + & + & + & + & & . & $\cdot$ & & + & & + & & & + & + & & + & + & & & & & + & + & + & + & + & & & + \\
\hline & & on & & & . & & & & & . & $\cdot$ & & & & & & . & + & & & + & $\cdot$ & & & 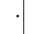 & . & & + & & & + & & & + \\
\hline & 를 & & 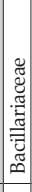 & 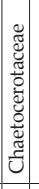 & 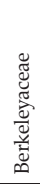 & 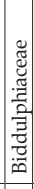 & 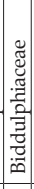 & 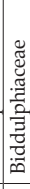 & 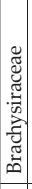 & 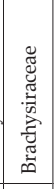 & 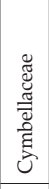 & 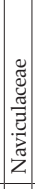 & 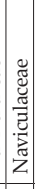 & 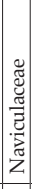 & 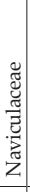 & 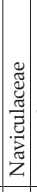 & 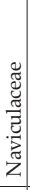 & 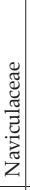 & 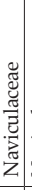 & 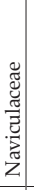 & 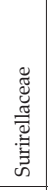 & 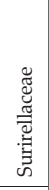 & 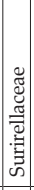 & 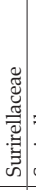 & 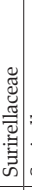 & $\begin{array}{l}\stackrel{\mathscr{\Xi}}{\mathbb{Z}} \\
\stackrel{\Xi}{\approx}\end{array}$ & 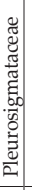 & 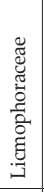 & & 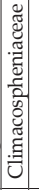 & 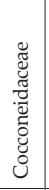 & 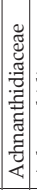 & 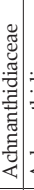 & 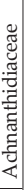 \\
\hline & 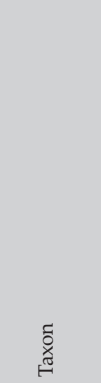 & & 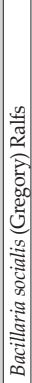 & 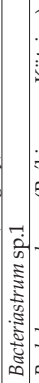 & 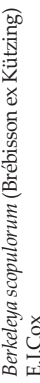 & 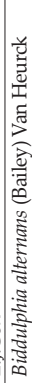 & 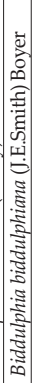 & 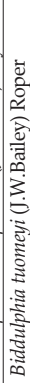 & 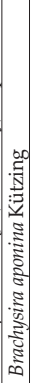 & 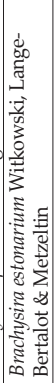 & 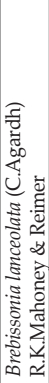 & 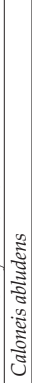 & 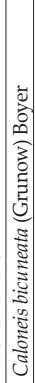 & 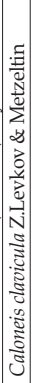 & 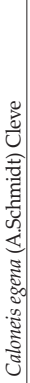 & 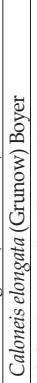 & 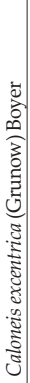 & 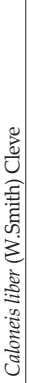 & 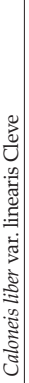 & $\begin{array}{c} \\
-1 \\
0 \\
\text { के } \\
.3 \\
\frac{3}{3} \\
\frac{3}{3}\end{array}$ & 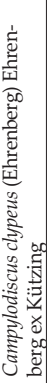 & 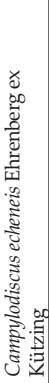 & 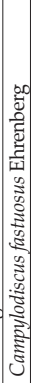 & 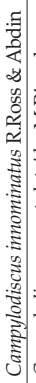 & 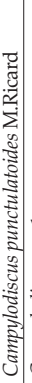 & 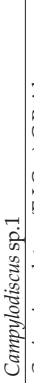 & 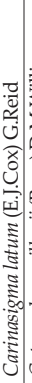 & 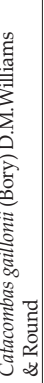 & 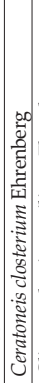 & 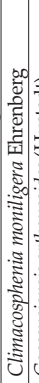 & 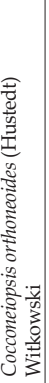 & 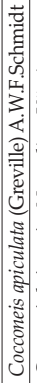 & 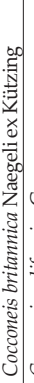 & \\
\hline
\end{tabular}




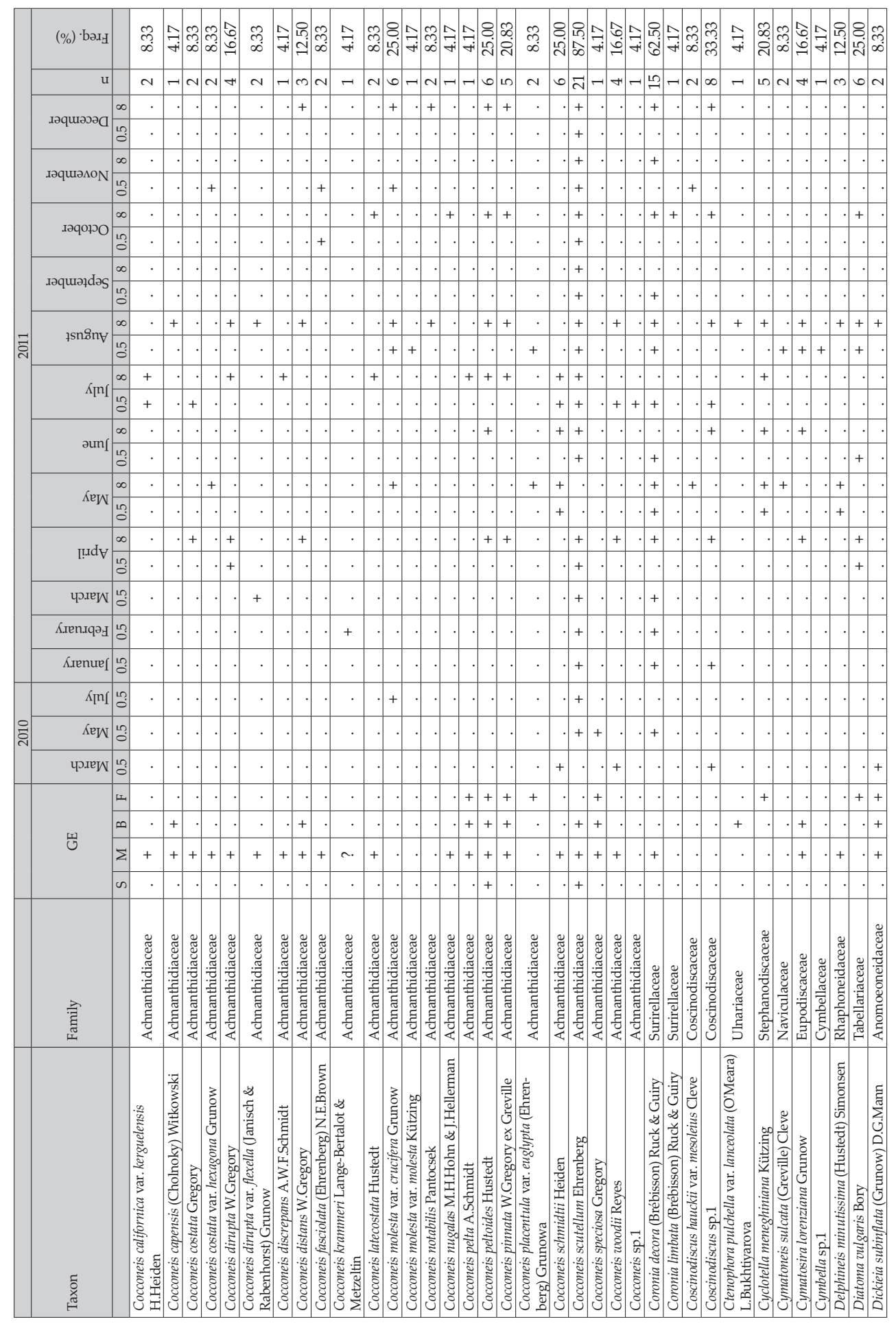




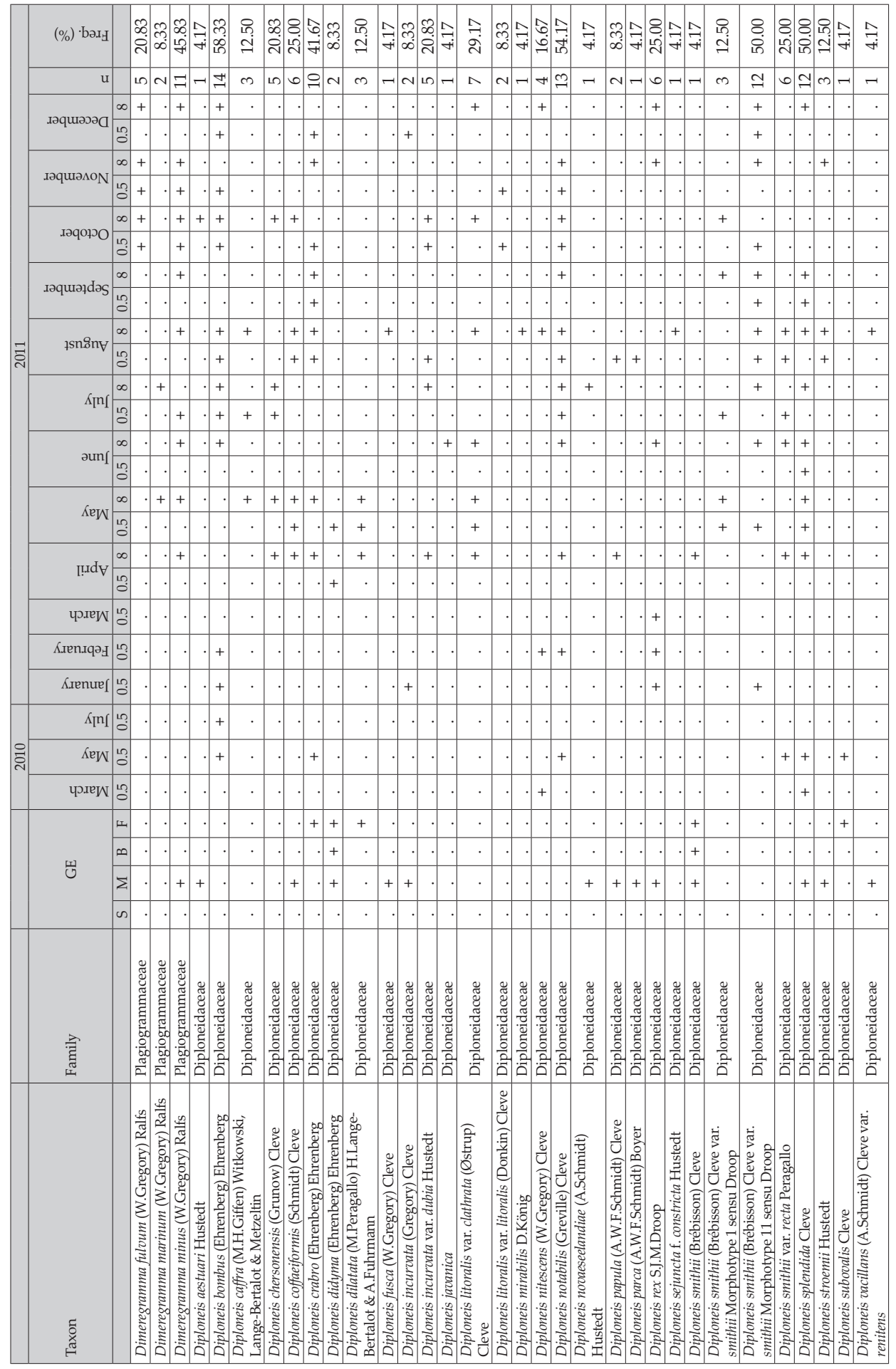




\begin{tabular}{|c|c|c|c|c|c|c|c|c|c|c|c|c|c|c|c|c|c|c|c|c|c|c|c|c|c|c|}
\hline (\%) bəə, & 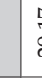 & îे & & d) & & $\vec{F}$ & 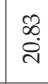 & $\begin{array}{c}\infty \\
\infty \\
\infty\end{array}$ & & 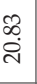 & $\overrightarrow{7}$ & 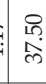 & & & $\vec{f}$ & $\stackrel{\nearrow}{F}$ & & & $\stackrel{7}{\rightarrow}$ & Fे & $\overrightarrow{+}$ & $\stackrel{F}{F}$ & & & & $\begin{array}{lll}\circ \\
0 \\
0\end{array}$ \\
\hline & & A & m. & 0 & $N-$ & - & n & $N$ & & in & $-1-$ & $a$ & & 4 & $-1-$ & - & H & & -1 & $r$ & -1 & -1 & in - & | & $\infty \leq 5$ & $=m$ \\
\hline 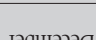 & $\infty$ & & & & + & 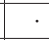 & $\cdot$ & & + & . & & • & & & & . & $\cdot$ & & & $\cdot$ & . & . & 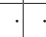 & + & t &.+ \\
\hline (1) & ¿? & & & & & . & . & + & & $\cdot$ & & + & & & & . & . & & & . & $\cdot$ & $\cdot$ & + & + & + & + . \\
\hline тәдшәА૦N & $\infty$ & & & & + & . & & & + & + & & + & . & . & &. & + & & . & $\cdot$ & . & . & 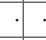 & + & + &.+ \\
\hline & เి & & & & & . & + & & & . & . 1 & + & . & & & . & + & & & . & & . & & & + & . \\
\hline & $\infty$ & + & + &. & . &. & + & $\cdot$ & & $\cdot$ &. & . & + & $\cdot$ & ${ }^{\circ}$ &. & + & & tra & $\cdot$ & $\cdot$ & . & . & + &.+ & . \\
\hline . & ? & . & & + & & . & . & & & + & $\cdot$ & . & & & & . & t & & . & . & . & . & 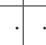 & & ++ & + . \\
\hline ع & $\infty$ & . & & + & & . & . & 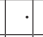 & 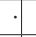 & $\cdot$ & $\cdot$ & . & o. & 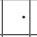 & . & . & $\cdot$ & & . & $\cdot$ & . & . & . & 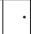 & + &.+ \\
\hline Iन & เి & . & & + & & . & . & & & $\cdot$ & & . & . & . & & . & $\cdot$ & & 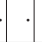 & $\cdot$ & & . & & 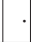 & + & + . \\
\hline fsnony & $\infty$ & + & & & $\cdot$ & . & + & + & + & + &.+ & + & . & . & . &. & . & & + & + & $\cdot$ & + & & + & ++ &.+ \\
\hline & 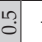 & + & & & 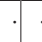 & . & . & & & + & & + & + & & & . & . & & & . & & . & 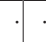 & 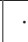 & ++ & + . \\
\hline & $\infty$ & + & & . & 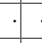 &. & + & & & $\cdot$ & + & . & . & i. & . & + & . & & & $\cdot$ & . & . & 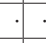 & + & $\cdot+$ & ++ \\
\hline & 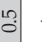 & + & & 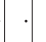 & 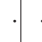 & . & . & & & $\cdot$ & & + & & & & . & . & & & $\cdot$ & . & . & & & ++ & + . \\
\hline כunf & $\infty$ & 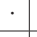 & & . & + & . & . & . & 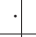 & $\cdot$ & . & + & . & . & + & . & $\cdot$ & & 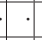 & . & $\cdot$ & . & . & + &.+ & ++ \\
\hline & ?ี? & & & + & & . & . & & & $\cdot$ & & . & & & & . & th & & & . & (1) & . & & & $\cdot{ }^{\circ}$ & . \\
\hline $\mathrm{S}_{\mathrm{S}}$ & $\infty$ & . & + & & + & + & . & & + & $\cdot$ & . & . & . & . & . & . & + & + & & . & $\cdot$ & . & 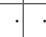 & 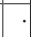 &. & . \\
\hline & เి & . & + & & 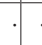 & . & . & & + & . & & . & & . & & . & & & & . & . & . & & 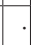 & $\cdot$ & . \\
\hline & $\infty$ & + & + & & t. & . & + & & & + & & + & & + & + & . & 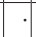 & & & . & + & . & + & 1 & ++ &.+ \\
\hline t. & 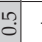 & + & & & + & . & 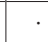 & & & $\cdot$ & & . & & & & . & 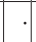 & & & . & & . & & & + & . \\
\hline чग्е & ?ำ & & & + & & . & . & & & $\cdot$ & & . & & & & . & 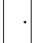 & & & . & & . & & & + & + . \\
\hline K.ensqa & ?? & . & & & & . & . & & & $\cdot$ & & . & & & & . & 1 & & & $\cdot$ & & . & + & & + & + \\
\hline Krenuef & है & & & & & . & . & + & & $\cdot$ & & + & & & & . & 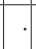 & & & . & & . & + & + & + & + . \\
\hline${ }_{\mathrm{K}}^{\mathrm{I}} \mathrm{n} \mathrm{f}$ & เి & & & & & . & . & & & $\cdot$ & & . & & & & . & & & & . & & . & 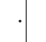 & & . & . \\
\hline${ }^{K e} \mathrm{~W}$ & เి & & & & 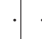 & . & . & & & . & & . & & & & . & & & & . & & . & + & & 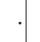 & . \\
\hline yрхек & ?อ & & & & + & . & . & & & $\cdot$ & & . & & + & & . & & + & & . & & . & + & & 7 &.+ \\
\hline & 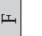 & & & & + & . & + & & & + & $\cdot$ & . & & $\cdot$ & . & . & & & & + & + & + & & 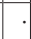 &. &. \\
\hline & $\infty$ & . & + & & & $\cdot$ & + & & + & + & ++ & + & & & . & . & & & & $\cdot$ & & . & & + & - &.+ \\
\hline 0 & $\Sigma$ & & + & & & + & + & & + & + & ++ & + & & & ++ & . & & & & . & & . & & + &.+ & ++ \\
\hline & is) & & + & & & & & . & + & + & + & . & & $D^{2}$ & + & . & & & & . & & . & & & $\cdot 1$ &.+ \\
\hline 屁 & 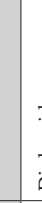 & . & 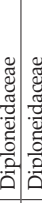 & & 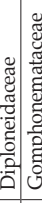 & 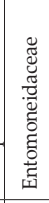 & 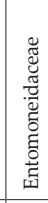 & 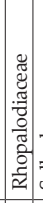 & & 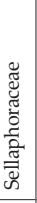 & 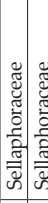 & 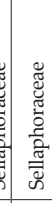 & 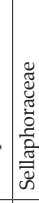 & 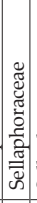 & 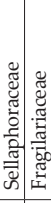 & 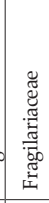 & 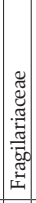 & 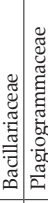 & 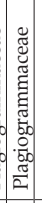 & 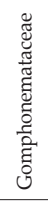 & 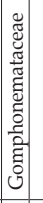 & 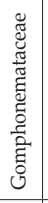 & & & 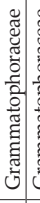 & 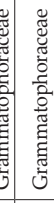 \\
\hline $\begin{array}{l}\text { 突 } \\
\text { 胥 }\end{array}$ & 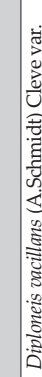 & $\frac{1}{2}$ & 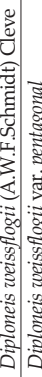 & 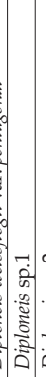 & 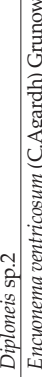 & & 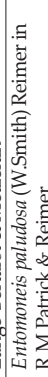 & 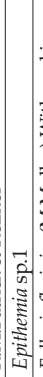 & $\frac{3}{2}$ & 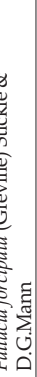 & 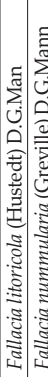 & 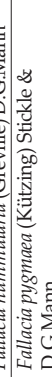 & 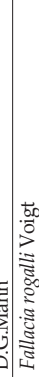 & 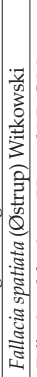 & 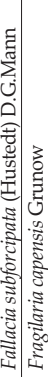 & 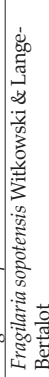 & 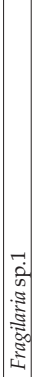 & 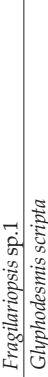 & & 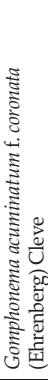 & 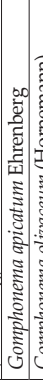 & 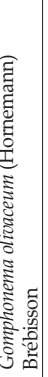 & 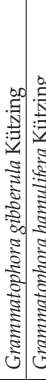 & & 部 & 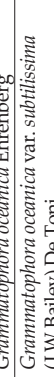 \\
\hline
\end{tabular}




\begin{tabular}{|c|c|c|c|c|c|c|c|c|c|c|c|c|c|c|c|c|c|c|c|c|c|c|c|c|c|c|c|c|c|c|c|c|c|c|}
\hline & (\%) $\cdot$ bəa & & $=$ & 7 & 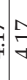 & 7 & : & & $\overrightarrow{+}$ & 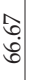 & & 它: & & & & 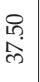 & $\begin{array}{c}m \\
\infty \\
\infty \\
\infty\end{array}$ & $\begin{array}{l}\text { in } \\
\text { in } \\
-1\end{array}$ & 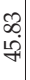 & $\begin{array}{c}\infty \\
\infty \\
\infty \\
\infty\end{array}$ & 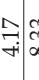 & $\begin{array}{c}\infty \\
\infty \\
\infty \\
\infty\end{array}$ & $\begin{array}{l}\infty \\
m \\
\infty \\
\infty\end{array}$ & 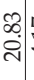 & & & $\hat{i}: \overrightarrow{+}$ & 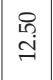 & $\underset{+}{\stackrel{A}{*}}$ & 志 & ○: & $\overrightarrow{+}$ & $\stackrel{\triangle}{\stackrel{ }{+}}$ & ลे \\
\hline & & & -1 & -1 & -1 & -1 & $m$ & 10 & -1 & ?. & in & + & -1. & -1. & -1 & $a$ & $N$ & $\infty$ & $=$ & $N$ & -1 & $\mathrm{~N}$ & $N$ & 10 & -1 & r & $v-$ & $m$ & -1 & 9 & 6 & - & -1 & $\Lambda$ \\
\hline & & $\infty$ & + & + & & & & & & + & & & & + & & . & & & & & & & $\cdot$ & & & & + & . & . & + & & & . & \\
\hline & & | & & & & + & & & & + & & & & & & . & & & & & & & . & & & & & . & $\cdot$ & + & & & . & \\
\hline & & $\infty$ & & & & & & + & & t & & & . & & & . & & & + & & & & . & & & + & & $\cdot$ & + & + & & & . & \\
\hline & & เొ & & & & & & & & + & & & & & & . & & & & & & & . & & & & & + & & . & & & . & \\
\hline & & $\infty$ & & & & & & . & & + & + & & & & & + & & & + & & & & . & & & & & . & & . & & & . & \\
\hline & & In: & & - & & & & . & & + & & $\cdot$ & $\cdot$ & & & $\cdot$ & & & 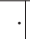 & $\cdot$ & & & - & . & & & & - & & . & & & $\cdot$ & \\
\hline & wädac & $\infty$ & & & & & & . & & 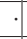 & & . & $\cdot$ & & & $\cdot$ & & & + & & & & $\cdot$ & . & & & & . & & + & & & . & \\
\hline & & 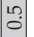 & & & & & & . & & + & & $\cdot$ & . & & & $\cdot$ & & & + & & & & . & $\cdot$ & & & & . & & + & & & . & 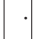 \\
\hline & 3 ny & $\infty$ & & & & & & . & & + & + & + & + & & & + & & 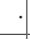 & + & 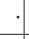 & & & . & + & & + & & $\cdot$ & & + & & & . & + \\
\hline & & เి & & & & & & . & & + & + & . & & & & + & & & $\cdot$ & 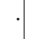 & & . & . & $\cdot$ & & & & . & & + & & & . & + \\
\hline & & $\infty$ & & & & & & + & & + & + & + & & & & + & & + & + & & & + & + & + & & & & . & & + & & & $\cdot$ & + \\
\hline & & ก & & & & & & 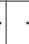 & & + & & . & & & & + & & & $\cdot$ & 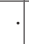 & & & + & & & & & $\cdot$ & & + & & &. & . \\
\hline & & $\infty$ & & & + & & 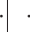 & 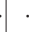 & & . & & + & & & & + & + & & + & . & & . & . & . & $\cdot$ & & & $\cdot$ &. & . & & . & $\cdot$ & + \\
\hline & & $\stackrel{2}{0}$ & & & & & + & + & & $\cdot$ & & & & & & . & & & + & 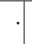 & & . & . & & & & & - & & - & & & - & \\
\hline & Spot & $\infty$ & & & & & & 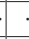 & & + & . & & & & & + & & & + & 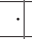 & & + & · & t & & & & . & & + & + & & . & . \\
\hline & & เి & & & & & & + & & + & + & & & & & - & & & 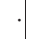 & ${ }^{\circ}$ & & & . & 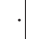 & & & & . & & . & + & + & - & . \\
\hline & & $\infty$ & & & & & & & & + & & & & & + & + & & + & . & . & & & . & + & & & & + & & + & + & & . & + \\
\hline & & |ก: & & & & & & . & & + & & & & & . & · & & & $\cdot$ & . & . & & - & & & & & + & & + & & & . & + \\
\hline & чрле W & ก & & & & & + & & + & & & & & & + & . & & & + & + & + & & . & & & & & . & & 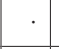 & & & 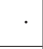 & \\
\hline & Кrentqән & เి & & & & & & 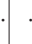 & & + & & + & & & & + & & + & & & & & . & + & & & & . & & . & + & & + & \\
\hline & Krenuef & เి & & & & & + & & & + & & & & & & . & & & & & & & . & & & & & . & & . & & & • & \\
\hline & $\mathrm{s} n \mathrm{~ns}$ & $\stackrel{2}{\circ}$ & & & & & & & & & & & & & & . & & & & & & & - & & & & & . & & & & & . & \\
\hline & eW & เి & & & & & & + & & & & & & & & . & + & & & & & & . & & & & & . & & + & + & & . & \\
\hline & IeW & เి & + & & & & & & & & & & & & & . & & & + & . & & & . & + & + & + & & . & & . & + & & . & + \\
\hline & & 功 & & & & & & . & . & & & $\cdot$ & & . & . & . & & & . & + & & & + & & . & & & . & & . & & . & $\cdot$ & . \\
\hline & & $\infty$ & & & & & & + & + & & & 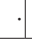 & & + & + & . & & & + & + & & . & + & + & . & & & . & & . & & + & $\cdot$ & . \\
\hline & (u) & $\Sigma$ & + & & & & & + & & + & + & + & & + & & + & + & + & & & & & . & + & . & ++ & & + & & + & & & + & + \\
\hline & & os & & & & & & & & + & & & & + & & . & & & & & & & + & & & & & . & & . & & + & $\cdot$ & + \\
\hline & 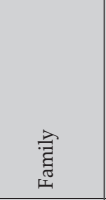 & & 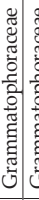 & 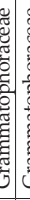 & 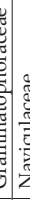 & 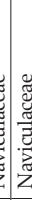 & 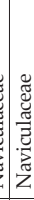 & & 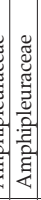 & 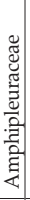 & 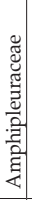 & 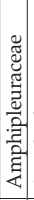 & 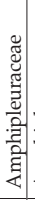 & 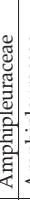 & 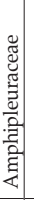 & 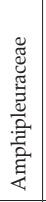 & 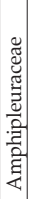 & 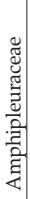 & 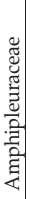 & 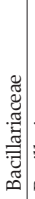 & 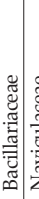 & 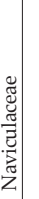 & 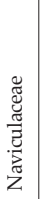 & 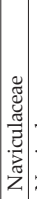 & 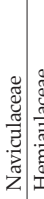 & 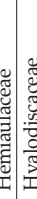 & 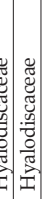 & 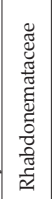 & 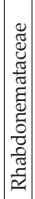 & 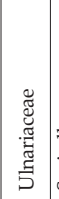 & 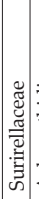 & 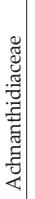 & 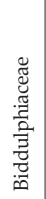 & 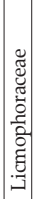 \\
\hline & 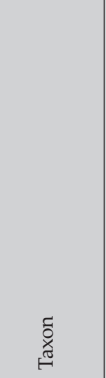 & & 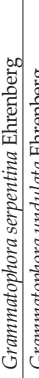 & . & 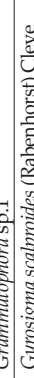 & |. & 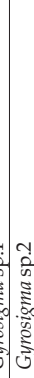 & 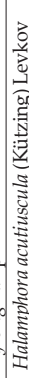 & 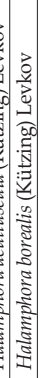 & 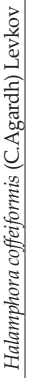 & 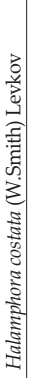 & 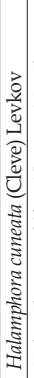 & 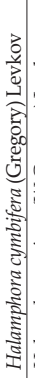 & 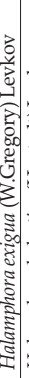 & 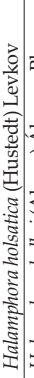 & 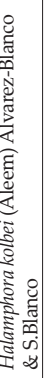 & 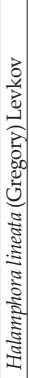 & 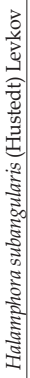 & 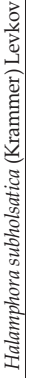 & 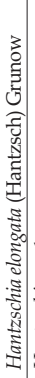 & 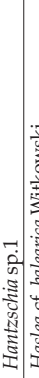 & 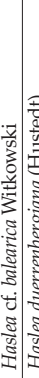 & 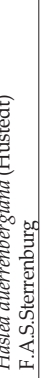 & 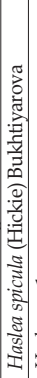 & 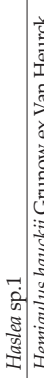 & 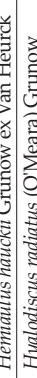 & 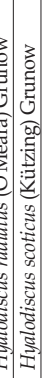 & 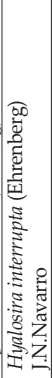 & 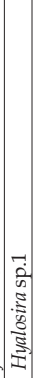 & 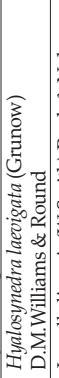 & 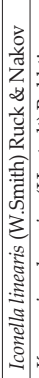 & 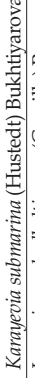 & 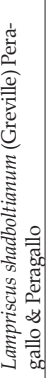 & 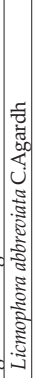 \\
\hline
\end{tabular}




\begin{tabular}{|c|c|c|c|c|c|c|c|c|c|c|c|c|c|c|c|c|c|c|c|c|c|c|c|c|c|c|}
\hline & $(\%) \cdot$ bə.t. & & 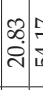 & & & & & & & & & $\underset{\infty}{\infty}$ & ن & 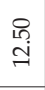 & & & 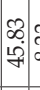 & $\infty$ & & 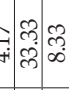 & & & & & ì & $z$ \\
\hline & & & inf & & $\infty$ & 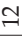 & & $t$ & - & \#- & -1 & $N$ & $H$ & $\infty$ & & $2-$ & $=$ & & & 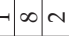 & $\omega m \rightarrow\llcorner$ & & $A \simeq$ & $y=m$ & & f \\
\hline & & $\infty$ & +- & & & & & & & + & &. & + & & + & + & + & & & + & & & + & + & & \\
\hline & & ¿? & & t & & & & & & & & & & & & & & & & & & 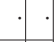 & . & & & \\
\hline & гәАО & $\infty$ & & & & & & & . & . & & $\cdot$ & & $\cdot$ & & + & + & & & + & + & + &. &. & $\cdot+$ & \\
\hline & & $\stackrel{2}{\circ}$ & & & & + & & + & & . & & . & & . &. & & . & + & & $\cdot$ & 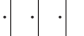 & & . & $\cdot$ & . & \\
\hline & 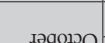 & $\infty$ & $\cdot$ & & & & & & $\cdot$ & 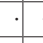 & & $\cdot$ & + & $\cdot$ & + & + & + & _. & $\cdot \cdot$ & +5 & $\cdot++$ &. & & ++ & + & \\
\hline & & ? & & & & & & & + & . & & $\cdot$ & & . & 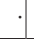 & + & + & + & & . & 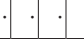 & . & + & + & . & \\
\hline & y-dast & $\infty$ &. & 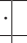 & & + & & & L & . & & $\cdot$ & & $\cdot$ & + & & + & + & $\cdot$. & $\cdot \cdot$ & $\cdot \cdot \cdot$ & $\cdot$ & + & 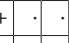 &. & \\
\hline & & ? & . & 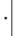 & & & & + & & + & & $\cdot$ & & . & + & + &. & + & + & + & 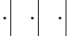 & . & + & + & . & \\
\hline & $1 \mathrm{sn} 8 \mathrm{ny}$ & $\infty$ & +- & $+t$ & & + & & + & & + & + & $\cdot$ & + & $\cdot$ & + & + & + & . & $\cdot \cdot \cdot$ & \begin{tabular}{l|l}
+ \\
\end{tabular} &. & ++ & ++ & $+\cdot \cdot \cdot$ & & + \\
\hline & & เి & +- & 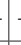 & & + & & & & & & $\cdot$ & & + & . & & + & + & &.++ &. & + &.+ & + & & \\
\hline & $\kappa_{\mathrm{K}}^{\mathrm{n}}$ & $\infty+$ & . & & & + & & & & . & & $\cdot$ & & $\cdot$ & + & ++ & + & 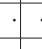 &. & ++ & + & ++ & + & + & & 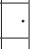 \\
\hline & & ?อ. & + & & & + & & & & & & . & & . & . & & 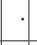 & + & & & + & 1 & + & + & & \\
\hline & zun $h$ & $\infty$ & & & & + & & & . & . & & $\cdot$ & & $\cdot$ & + & + &. & . & 7 & $\cdot$ & 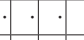 & $\cdot$ & + & + & - & 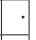 \\
\hline & & ? & -1 & $+t$ & & + & + & & & . & & . & & . & $\cdot$ & 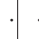 & . & . & & . & &. & + & & & \\
\hline & & $\infty$ & -1 & & & + & + & & & ++ & & + & & $\cdot$ & + & + & . & + & 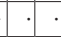 & . & $\cdot$ & $\cdot+$ & + & & & \\
\hline & & $\stackrel{1}{\circ}+$ & . & . & & & + & . & & & & . & + & & . & + & . & . & & . & &. &.+ & & & \\
\hline & & $\infty$ & + & . & & & & & &. & & + & & + & + & + & + & + & & + & + & ++ & $\cdot$ & & & \\
\hline & & เి & & 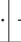 & & & + & & & & & 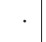 & & ${ }^{\circ}$ & $\cdot$ & & . & & & & & & $\cdot$ & & & \\
\hline & чग्ё & เి & & + & & + & & + & & & & $\cdot$ & & + & + & + & + & & & & & & + & + & & \\
\hline & K.леn.qә & : & 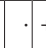 & + & 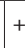 & + & & t & & & & . & & . & & & + & & & & & & + & + & & \\
\hline & enuer & เి & & & & & & & & & & · & & ${ }^{\circ}$ & & & & & & & & & & & & \\
\hline & ${ }^{\mathrm{K}}[\mathrm{n}[$ & $\stackrel{0}{0}$ & & 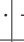 & & & & & & & & . & & . & & & & & & & + & & & & & \\
\hline & KeW & เి + & 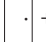 & + & + & & & & & & & . & & . & & & & & & & & & & & & \\
\hline & ציקיא & ? & 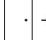 & 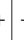 & & & & 1 & & & & . & & . & & + & & + & & & & & & & & \\
\hline & & 4 & & & & & & & + & . & & . & & & & & t. & & & . & . & & & &. & \\
\hline & 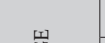 & $\infty$ & & & & & + & & & . & & . & & & & & . & & & . & + & 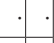 & & &. & \\
\hline & 0 & $\Sigma \mid+$ & + & . & & & & & & + & & + & & & + & & + & & & + & 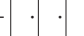 & + & & + & & + \\
\hline & & os & & & & & & & & + & & & & - & + & & + & & & & & & & & & \\
\hline & है & 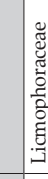 & 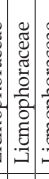 & & & & & & & & & 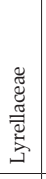 & 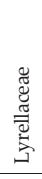 & 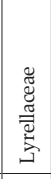 & $\mid$ & & . & & 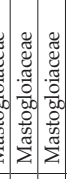 & 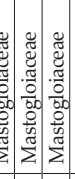 & 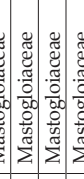 & 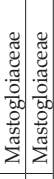 & 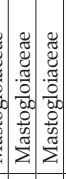 & 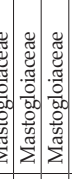 & 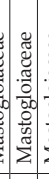 & 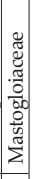 \\
\hline & $\begin{array}{l}\text { ర్ } \\
\end{array}$ & : & 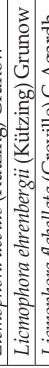 & & & & & & $\mid$ & 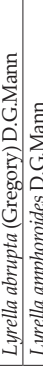 & 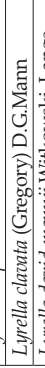 & 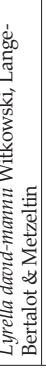 & 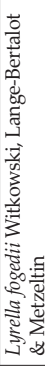 & 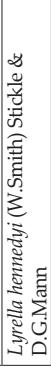 & $\mid$ & |c. & 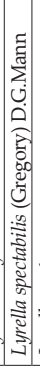 & 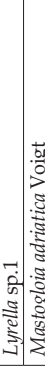 & 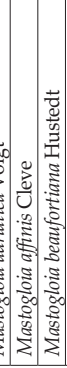 & 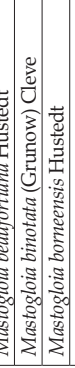 & 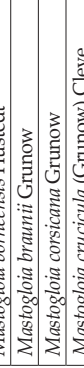 & 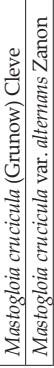 & 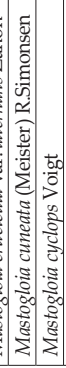 & 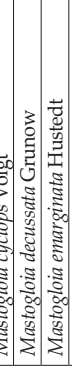 & 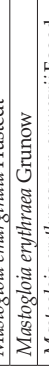 & 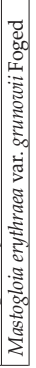 \\
\hline
\end{tabular}




\begin{tabular}{|c|c|c|c|c|c|c|c|c|c|c|c|c|c|c|c|c|c|c|c|c|c|c|c|c|c|c|c|c|c|c|c|c|c|c|c|c|}
\hline & (\%) $\cdot$ bән & & 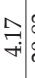 & & & & & & & & ְُ & & : & 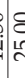 & & 8 & & & $\begin{array}{c}\infty \\
\infty \\
\infty\end{array}$ & 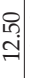 & & $\vec{\partial}$ & & $\begin{array}{c}\infty \\
\infty \\
\infty\end{array}$ & & & & & בְּ & & $=\frac{\hat{\sigma}}{\dot{q}}$ & $\stackrel{\infty}{\infty}$ & $\ddot{\infty}$ & & 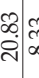 & ल⿸户 \\
\hline & & & -1 & in & เ & o. & 12 & م. & $\infty=$ & $\exists \mathrm{c}$ & 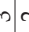 & w & $+\mathrm{Cr}$ & 6 & $m$ & & $N$ & $m$ & $N$ & $\infty$ & $m$ & $\triangle$ & N & N. & -1 & $-1 \mathrm{c}$ & No & & $m 1$ & & -10 & in & $N$ & & in 0 & N \\
\hline & & $\infty$ & & & + & + & & & . & + & & & & + & & & & . & & & & . &. & & + & & & & + & & + & & . & & & \\
\hline & & |n: & & & & & & & & & & + & & & & & &. & & & & . & $\cdot$ & & & & & & & & . & . & + & & & \\
\hline & & $\infty$ & & + & & & & . & + & + & & & & + & & & & . & & & & . & & & & & & + & + & & . & & . & & & \\
\hline & & : & & & & & & . & + & + & + & & & & . & & $\cdot$ & 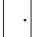 & 1 & . & & . & $\cdot$ & & . & & & ++ & + & & + & & . & & . & \\
\hline & & $\infty$ & + & & + & + & & + & + & + & & & & + & + & & + & & + & + & + & + & + & & & & & $\cdot$ & + & & + & & $\cdot$ & & & \\
\hline & 400 & "ก: & & & & & & + & + & . & & & & + & & & & $\cdot$ & & & & - & & & $\cdot$ & & & ++ & + & & + & . & $\cdot$ & & & \\
\hline & ch & $\infty$ & & + & & & & $\cdot$ & + & . & & & 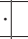 & + & & & & . & $\cdot$ & & . & . & $\cdot$ & 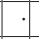 & & & & + & 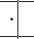 & & $\cdot \cdot$ & . & $\cdot$ & & & \\
\hline & & $\stackrel{102}{0}$ & & & . & + & + & . & + & + & . & & + & & & & & $\cdot$ & & $\cdot$ & & + & & + & & & & + & + & & + & . & . & & & \\
\hline & מע & $\infty$ & & + & + & + & & 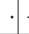 & + & + & . & 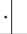 &. & 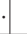 & + & & $\cdot$ & . & . & + & & + & $\cdot$ & & & + & ++ & + & + & & + & & . & & . & \\
\hline & & : & & & + & & & + & 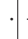 & + & . & + & & & & & . & + & . & $\cdot$ & . & + & . & . & & & & + & . & & + & + & . & & + & \\
\hline & & $\infty$ & & + & & + & & + & . & +1. & + & & & & & & + & + & + & + & & . & + & + & & & & + & + & & . & ${ }^{\circ}$ & . & & & \\
\hline & & : & & & & ${ }^{\circ}$ & & + & + & + & & & & & & & & $\cdot$ & & & . & + & & & & & 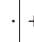 & + & 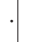 & & + & 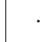 & . & & & \\
\hline & & $\infty$ & & & & + & & & & $\cdot-$ & + & & + & & & & & $\cdot$ & & & & + & & & & & + & & + & & + & ${ }^{\circ}$ & $\cdot$ & & . & \\
\hline & & $\stackrel{12}{\circ}$ & & & . & & & & & . & & & & & & & & $\cdot$ & & & . & . & & & & & & & + & & + & + & . & & & \\
\hline & Spit & $\infty$ & & + & + & & & & & + & & & & & 1 & & & . & ${ }^{\circ}$ & & + & $\cdot$ & . & & & & & & & & & . & . & & + & + \\
\hline & & $\stackrel{100}{0}$ & & & & & & . & & . & . & 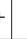 & & + & & & & . & & & + & . & $\cdot$ & & & & & & & & & + & . & & + & \\
\hline & & $\infty$ & & & & + & & & & + & & & + & & + & & & + & 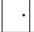 & & & . & $\cdot$ & & & & & & & & & 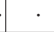 & 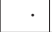 & & & + \\
\hline & & : & & & & & & & & & & & & & & & & & & & & . & & & & & & & & & & . & . & & & \\
\hline & IeW & ำ & & & & & & & & & & + & & & & & & & & & & + & & & & & & & & & & & 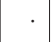 & & & \\
\hline & 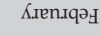 & 눙 & & & & & & & & & & + & & & & & & & & & & . & & & & & & & & & & & . & & + & \\
\hline & Krenue & : & & & & & & & & & & & & & & & & & & & & . & & & & & & & & & & & r. & & & \\
\hline & $S_{\mathrm{J}} \mathrm{nf}$ & 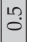 & & & & + & & & & & & & & & & & & & & & & . & & & & & & & & & & & & & & \\
\hline & $\mathrm{W}$ & 10 & & & & + & & & & & & & & & & & & . & . & & & . & & & & & & & & & & + & + & & & \\
\hline & IeW & : & & & & & & & & & & & & & & & & & & & & . & & & & & & & & & & + & $\cdot$ & & + & \\
\hline & & $\Phi$ & & & & & & & & & & & & & & & & . & . & $\cdot$ & $\cdot$ & . & & + & & & & + & + & & & & . & & + & + \\
\hline & & $\infty$ & & & & & & & & . & & & & & & & & . & . & . & & . & & + & & & & + & + & & & + & + & & & \\
\hline & U & $\Sigma$ & & + & & + & & . & + & & & & + & ++ & + & & & + & + & + & & . & + & + & & & & & + & & & + & . & + & & \\
\hline & & os & + & & & & & & & & & & & & & & & & & . & & . & + & + & & & & + & + & & & & . & + & & \\
\hline & 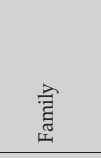 & & 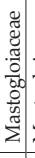 & 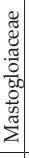 & 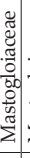 & . & 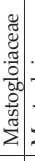 & 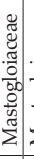 & 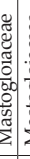 & 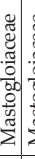 & 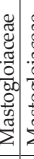 & 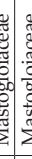 & 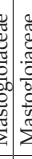 & 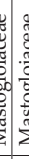 & 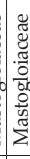 & 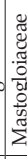 & 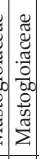 & 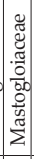 & 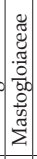 & 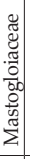 & 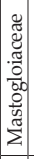 & 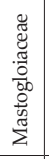 & 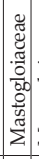 & 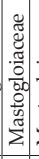 & 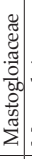 & 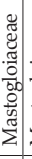 & 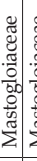 & 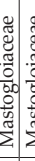 & 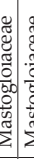 & 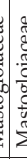 & 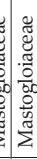 & 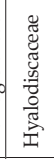 & 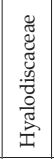 & 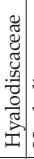 & 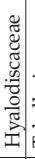 & 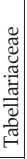 \\
\hline & 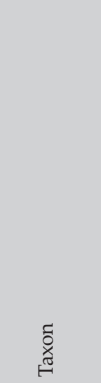 & & 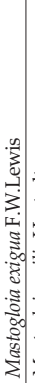 & 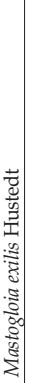 & 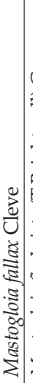 & 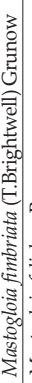 & 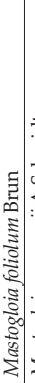 & 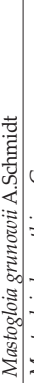 & 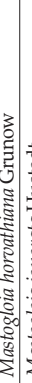 & 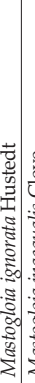 & 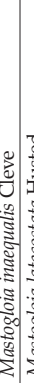 & 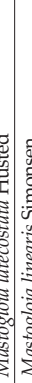 & 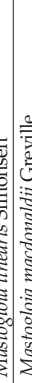 & 5 & 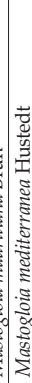 & 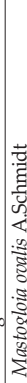 & 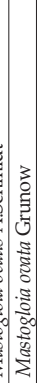 & 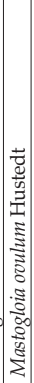 & 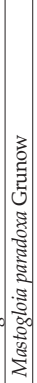 & 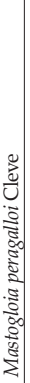 & 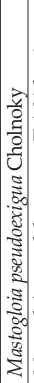 & 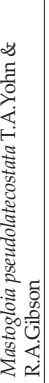 & 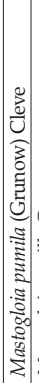 & 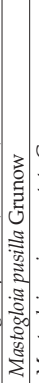 & 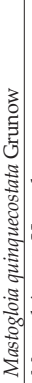 & . & 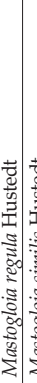 & 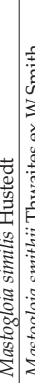 & 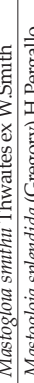 & $\frac{1}{2}$ & 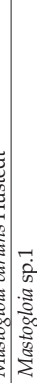 & 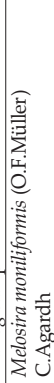 & 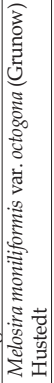 & 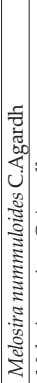 & 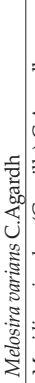 & 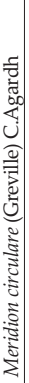 \\
\hline
\end{tabular}




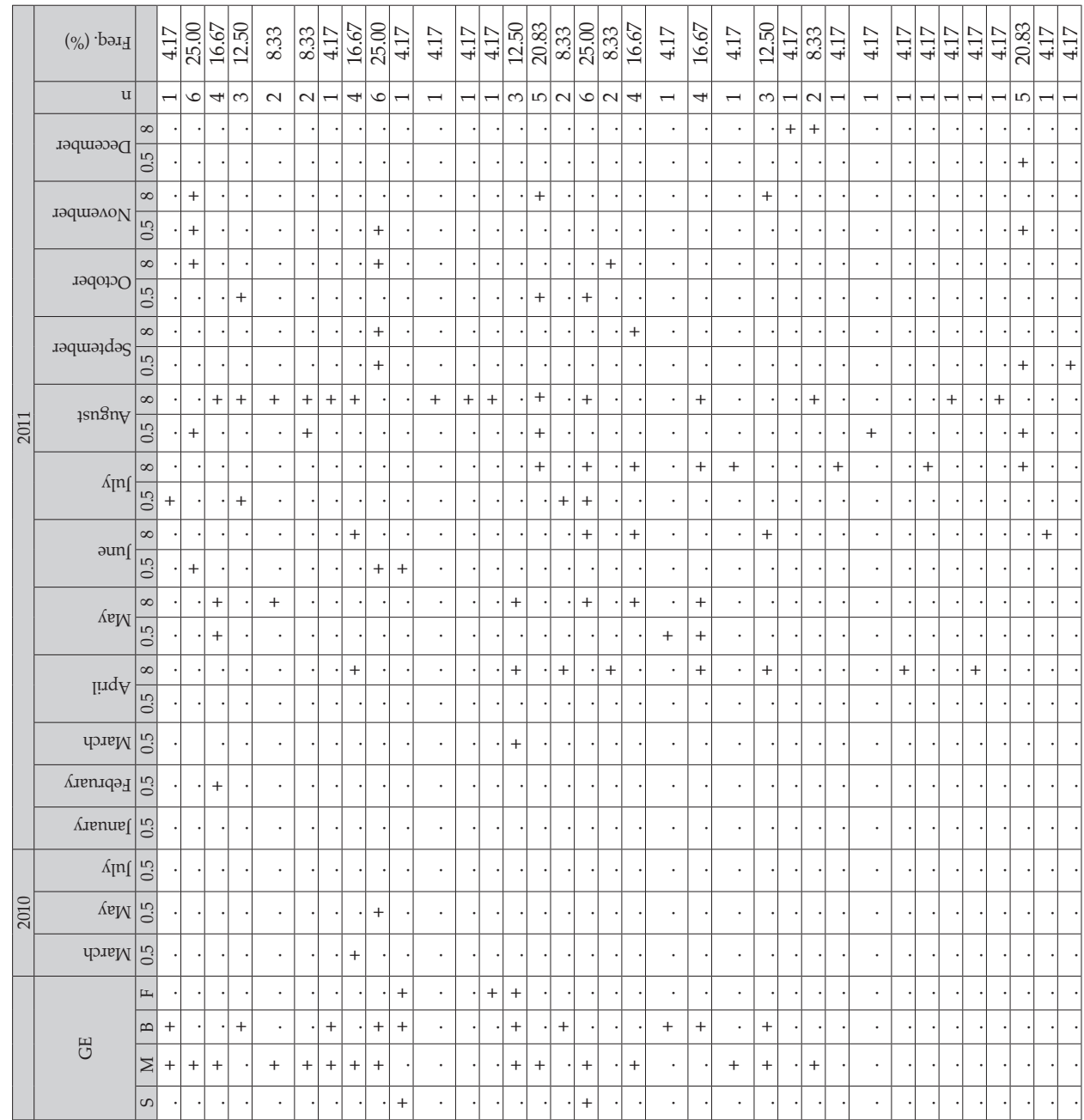

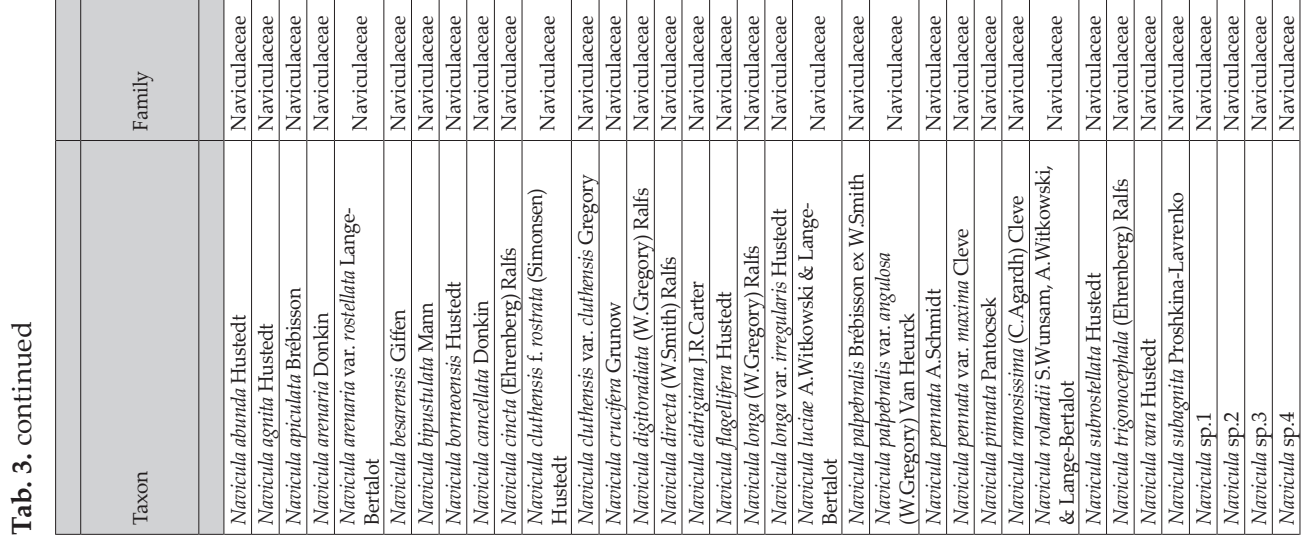




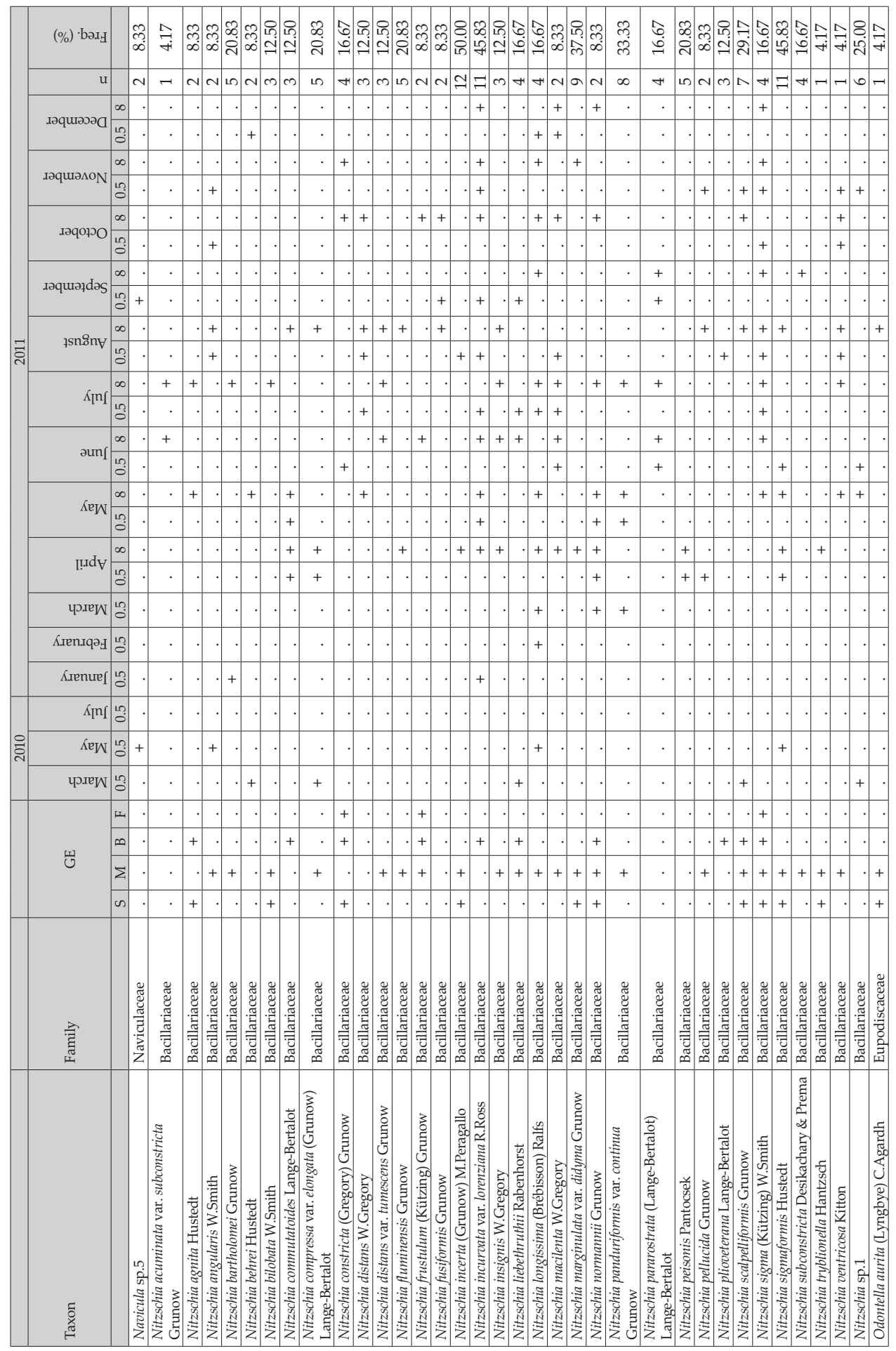




\begin{tabular}{|c|c|c|c|c|c|c|c|c|c|c|c|c|c|c|c|c|c|c|c|c|c|c|}
\hline 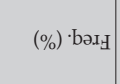 & $\begin{array}{c}\infty \\
0 \\
\infty \\
\infty\end{array}$ & $\begin{array}{c}\text { m } \\
\infty \\
\infty\end{array}$ & $\stackrel{亏}{\not}$ & $\begin{array}{l}\infty \\
\infty \\
\infty\end{array}$ & 구 & ف․ & $\vec{F}$ & 令 & $\aleph_{\infty}^{\infty}$ & $\begin{array}{c}\infty \\
\infty \\
\infty\end{array}$ & $\begin{array}{c}\infty \\
\infty \\
\infty\end{array}$ & \begin{tabular}{c|c}
8 & 8 \\
ț் & 8 \\
\end{tabular} & $\vec{D}+\vec{d}$ & 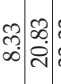 & $\begin{array}{lll}0 & 0 \\
0 & 0 \\
c & 0\end{array}$ & $\begin{array}{l}0 \\
\stackrel{0}{0} \\
\stackrel{0}{\circ}\end{array}$ & 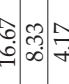 & 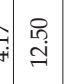 & $\mid$\begin{tabular}{ccc|}
$\infty$ & $\infty$ \\
$\infty$ & $\infty$ & 0 \\
$\infty$ & $\infty$
\end{tabular} & $\stackrel{\overline{+}}{+}$ & 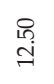 & 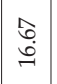 \\
\hline $\mathrm{u}$ & N & N & $r$ & $N$ & - & H & 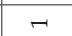 & m & N & N & 4 & \begin{tabular}{|l|l|}
0 & 0 \\
\end{tabular} & 9 & $N$ ம & \begin{tabular}{|l|l}
$\infty$ & - \\
\end{tabular} & H & $A N-$ & $n$ & $4 n$ & $r$ & $m$ & H \\
\hline & $\infty+$ & & . & & & & & & & & & + & + & & + & & & . & & & + & \\
\hline & | & & & 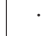 & & & & & & & & & & & + & & & . & + & & . & \\
\hline 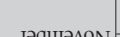 & $\infty$ & & . & . & & & . & & & & & + & + & & $\cdot$ & . & i & $\cdot$ & $\cdot$ & 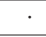 & . & . \\
\hline (2) & เి & & & . & & & . & & & & & + & + & & + & & & . & . & 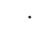 & . & . \\
\hline & $\infty$ & & . & . & & & . & + & & + & + & + & + & & + & & $\cdot$ & + & + & + & + & \\
\hline 4 & เి & . & . & . & & . & . & 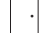 & . & & & . & + & & 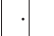 & . & $\cdot 1$ & $\cdot$ & $\cdot$ & . & . & . \\
\hline เ & $\infty$ & . & $\cdot$ &. & & . & . & . & . & & & . & $\cdot$ & . & . &. & + & + & 1 & . & . & $\cdot$ \\
\hline & ?: & ${ }^{\circ}$ & & $\cdot$ & & & . & + & & & & . & + & & 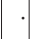 & . & . & $\cdot$ & $\cdot$ & . & . & \\
\hline 1engnyt - & $\infty$ & + & + &. & & + & . & & . & 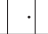 & . & + & ++ & + & ++- & + & + & + & 1 & . & . & \\
\hline 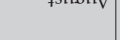 & t?: & . & $\cdot$ & . & & . & . & & . & & & + & . & & - & + & $\cdot 1$ & $\cdot$ & $\cdot$ & . & . & . \\
\hline & $\infty$ & . & $\cdot$ & + & & & $\cdot$ & $\therefore$ & + & t & & \begin{tabular}{|l|l|}
+ & + \\
\end{tabular} & + & 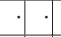 & 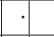 & 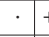 & ++ & $\cdot$ & t & . & . & + \\
\hline & : & . & & 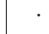 & & • & . & & & + & & & + & & 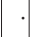 & $\cdot$ & 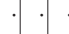 & $\cdot$ & 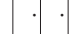 & 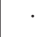 & . & . \\
\hline วй & $\infty$ & . & . & + & & $\cdot$ & . & t & + & & r. & . & + & & + & . & $+\cdot+$ & . & $\cdot 1$ & $\cdot$ & . & . \\
\hline & हैอ & . & & . & & . & . & & & & & $\cdot$ & + & & + & . & . & $\cdot$ & $\cdot \cdot$ & t & . & \\
\hline$s_{p}$ & $\infty+$ & + & $\cdot$ & . & & + & . & & & & & & ${ }^{\circ}$ & + & ++-1 & + & + & $\cdot$ & + & . & . & \\
\hline & เุ? & . & . & . & & + & . & & & & . & $\cdot$ & & $\cdot$ & & . & & . & & . & . & . \\
\hline udyt & $\infty$ & . & $\cdot$ &. & & + & . & + & $\cdot$ & & . & + & . & + & & + & & $\cdot$ & 1 & $\cdot$ & . & . \\
\hline & | & . & . & . & & . & + & & . & & + & + & . & 1 & & . & & $\cdot$ & 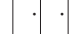 & . & . & . \\
\hline чргет & है & . & . & . & & . & . & & . & & & . & + & + & & . & . & . & . & . & . & . \\
\hline Клеn.qә & : & . & . & . & & . & . & & . & & & & & & & & & . & & . & . & + \\
\hline Krenuer| & เด & . & . & . & & & . & & & & & & & & & & & . & & & + & \\
\hline $\mathrm{K}_{[\mathrm{n}} \mathrm{n} \mid$ & เి & . & . & 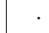 & & ${ }^{\circ}$ & ${ }^{3}$ & & . & & & & & + & & . & & . & 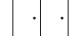 & . & 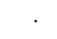 & . \\
\hline${ }^{\mathrm{K}} \mathrm{W}$ & & . & $\cdot$ & . & + & . & . & & . & & & + & 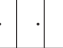 & + & & & . & $\cdot$ & + & . & . & + \\
\hline чग्रु & ? & . & . & . & & . & . & & . & & . & + & ++ &.+ & & . & . & . & . & $\cdot$ & . & + \\
\hline \multirow{4}{*}{0} & st & & + & + & & 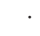 & . & & . & & $\cdot$ & + & . & $\cdot$ & 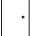 & . & + & $\cdot$ & . & · & . & . \\
\hline & $\infty$ & $\cdot$ & . & 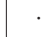 & & + & + & + & + & + & & & + & . & ++- & + & & $\cdot$ & . & . & . & . \\
\hline & $\Sigma$ & + & $\cdot$ & & + & + & + & + & + & $\begin{array}{lll}+ & \end{array}$ & + & & ++ & ++ & + & + & + & + & ++ & + & + & + \\
\hline & is & & . & $\cdot$ & + & & + & & + & + & & & + & & & + & & . & & & + & $\cdot$ \\
\hline 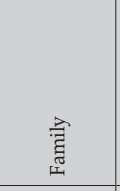 & 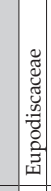 & 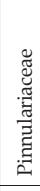 & 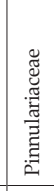 & 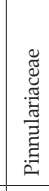 & & 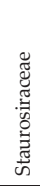 & 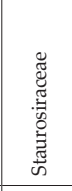 & 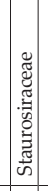 & 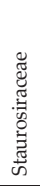 & 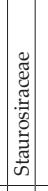 & 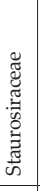 & 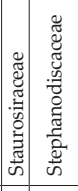 & 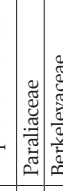 & 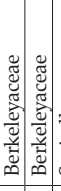 & 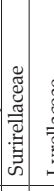 & 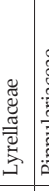 & 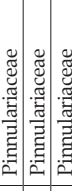 & 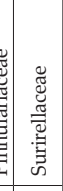 & 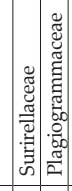 & 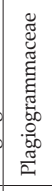 & 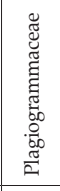 & 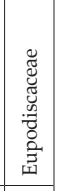 \\
\hline & 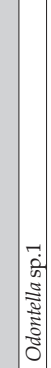 & 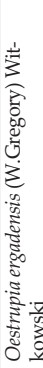 & 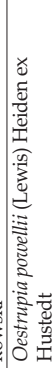 & 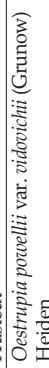 & & 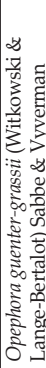 & 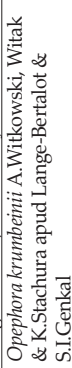 & : & 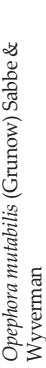 & 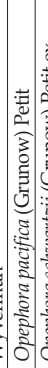 & है & 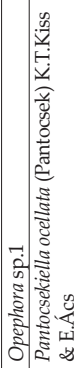 & 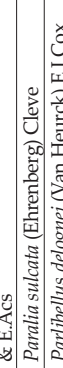 & 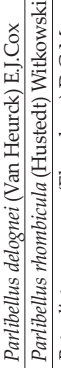 & 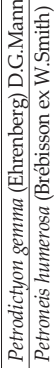 & 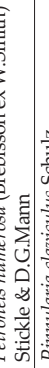 & 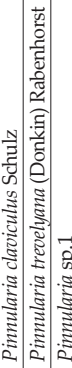 & 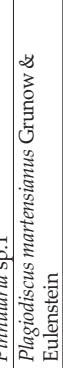 & 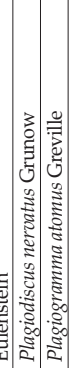 & 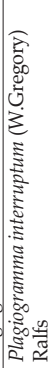 & 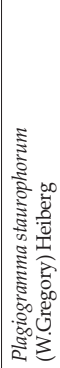 & 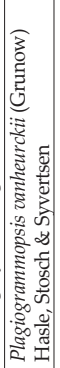 \\
\hline
\end{tabular}




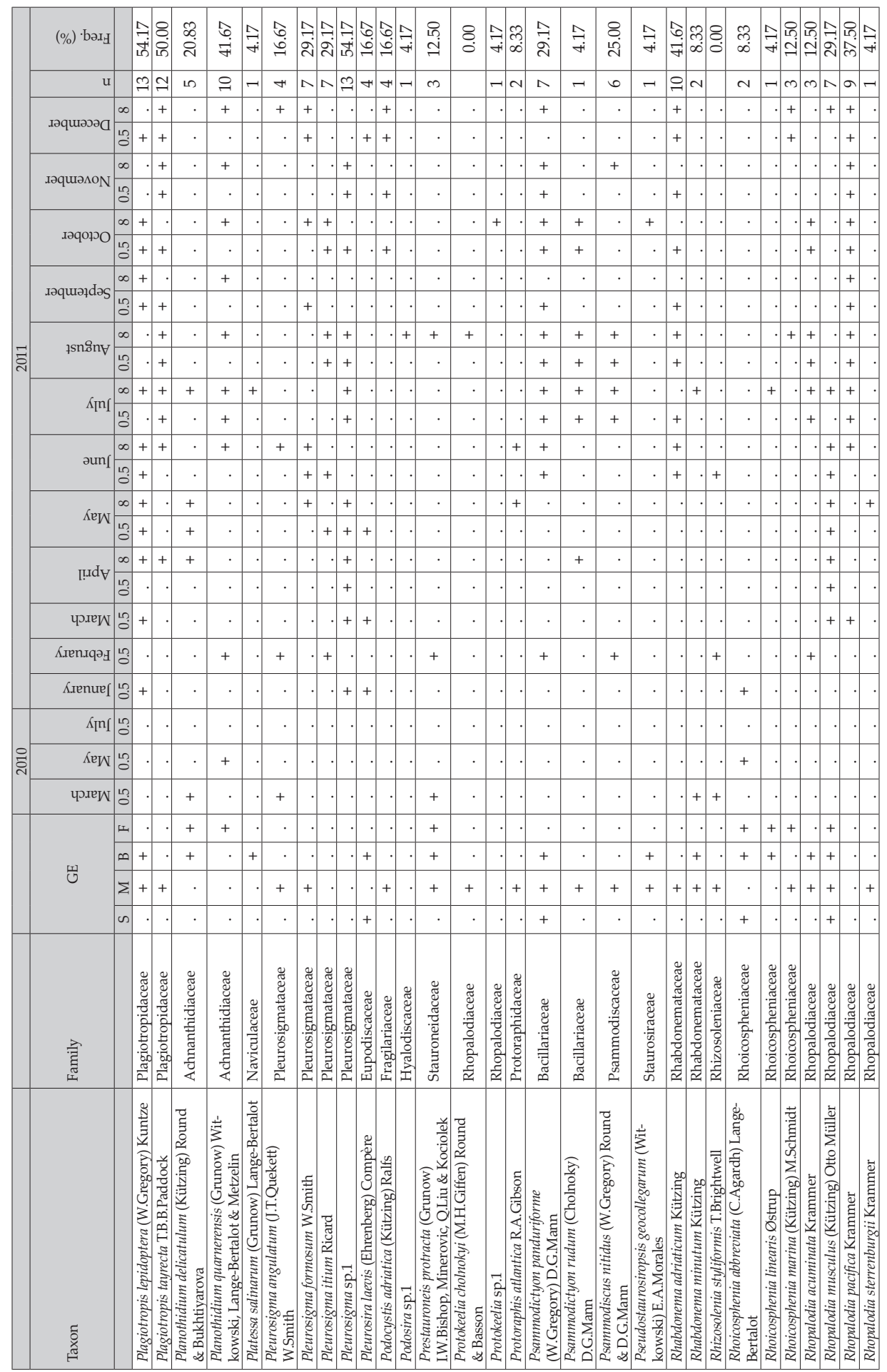




\begin{tabular}{|c|c|c|c|c|c|c|c|c|c|c|c|c|c|c|c|c|c|c|c|c|c|c|c|c|c|c|c|c|c|c|c|c|c|}
\hline & (\%) 'bə.. & & & $\stackrel{\vec{f}}{\vec{f}}$ & $\begin{array}{l}\infty \\
\infty \\
\infty\end{array}$ & 命 & $\stackrel{\vec{f}}{\vec{f}}$ & & & 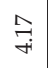 & $\stackrel{\overbrace{}}{\not}$ & $\begin{array}{c}\text { 足 } \\
\text { ஸे }\end{array}$ & & \begin{tabular}{|c|}
$\infty$ \\
$\infty$ \\
$\infty$ \\
$\infty$
\end{tabular} & & & & & & & 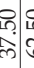 & 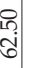 & $\begin{array}{c}m \\
\infty \\
\infty\end{array}$ & $\begin{array}{l}\stackrel{\leftrightarrow}{1} \\
\text { ำ }\end{array}$ & & $\stackrel{+}{7}$ & $\vec{a}$ & $\begin{array}{l}\hat{\sigma} \\
\stackrel{\emptyset}{0}\end{array}$ & $\begin{array}{l}\infty \\
\infty\end{array}$ & $\begin{array}{l}\infty \\
\infty \\
\infty\end{array}$ & ते. & & वे. \\
\hline & & & - & -1 & $N$ & t & - & $\Lambda$ & $=$ & - & -1 & $\sigma$ & 12 & $N$ & $\infty$ & $\infty$ & $m$ & -10 & N. & -10 & $\sigma \leq$ & 2 & $N$ & $m$ & $a$ & -1 & $\Lambda$ & + & $N$ & $N$ & $\triangle$ & 으: & 9 \\
\hline & & $\infty$ & & & . & & & & + & . & $\cdot$ & & + & & & + & & & + & & + & 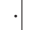 & $\cdot$ & $\cdot$ & + & & . & . & . & $\cdot$ & + & & + \\
\hline & & "20 & & & . & & & & + & . & $\cdot$ & & + & & & & & & & & + & + & $\cdot$ & $\cdot$ & & & . &. & . & $\cdot$ & & & + \\
\hline & & $\infty$ & & & . & & & & 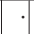 & + & . & & + & & & + & + & $\cdot$ & & & & & . & . & + & & . & . & . & $\cdot$ & . & & + \\
\hline & & เి & & & . & & & & + & . & $\cdot$ & & + & & & & & & & & & & . & $\cdot$ & & & . & + & . & . & + & & \\
\hline & & $\infty$ & & & . & & & + & . & $\cdot$ & $\cdot$ & & + & & + & $\cdot$ & . &. & . & & + & + & . & $\cdot$ & + & & + & + & . & . & & & + \\
\hline & & เุ้ & & & . & & & & + & $\cdot$ & $\cdot$ & & + & & & . & & . & & & & + & . & . & + & & . & $\cdot$ & + & $\cdot$ & & & . \\
\hline & & $\infty$ & & & $\cdot$ & & & . & $\cdot$ & $\cdot$ & $\cdot$ & & $\cdot$ & + & & + & • & $\cdot$ & . & & & + & $\cdot$ & $\cdot$ & . & 1 & . & $\cdot$ & . & $\cdot$ & . & & . \\
\hline & & น? & & & . & 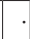 & & & + & $\cdot$ & $\cdot$ & & + & & & . & . & . & & & & + & $\cdot$ & . & & & . & $\cdot$ & . & . & . & & + \\
\hline & נsnin & $\infty$ & & . & $\cdot$ & + & 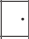 & . & . & . & $\cdot$ & & + & & . & + & + & $\cdot 1$ & 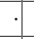 & 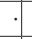 & & + &. & + & + & + & + & + &. & $\cdot$ & + & & + \\
\hline & & กै & & + & . & + & + & & + & $\cdot$ & $\cdot$ & & + & & & + & & & & & & + & . & $\cdot$ & 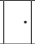 & & + & + & . & $\cdot$ & . & & + \\
\hline & Sm & $\infty$ & & & . & + & & & + & . &. & & + & $\cdot$ &. & $\cdot$ & & $\cdot$ & & + & + & $\cdot$ & . & $\cdot$ & + & & + & . & . & $\cdot$ & + & & + \\
\hline & & น? & & & . & & & & + & . & . & & + & & & + & & $\cdot$ & & & + & + & . & . & + & & . &. & . &. & & & + \\
\hline & & $\infty$ & & & + & & & & + & $\cdot$ & + & + & + & . &. & + & & $\cdot$ & . & & + & . & . & + & + & & $\cdot$ & $\cdot$ & . & $\cdot$ & . & & + \\
\hline & & เి & & & . & & & & + & . & $\cdot$ & & + & & 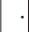 & & & $\cdot$ & & & & + & . & . & & & . & . & . & + & . & & + \\
\hline & & $\infty$ & + & & $\cdot$ & & & + & . & $\cdot$ &. & + & + & + & + & . & . & $\cdot$ & . & & + & + & . & $\cdot$ & ( & & + &. & . & $\cdot$ & + & + & + \\
\hline & $N$ & นึ? & & & . & & & + & & . & & + & + & & & & & $\cdot$ & & & + & + & - & $\cdot$ & & & + & . & . & . & + & + & + \\
\hline & & $\infty$ & & & . & & & + & . & . & . & + & . & . & $\cdot$ & $\cdot$ & + & + & & & . & . & + & $\cdot$ & + & & + & . & + & + & 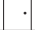 & + & + \\
\hline & & กั & & & . & & & + & & . & $\cdot$ & + & 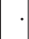 & & & . & & & & & . & . & . & $\cdot$ & & & . & . & 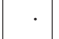 & . & 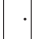 & + & + \\
\hline & чगге & 12 & & & . & ( & & + & & . & $\cdot$ & + & . & . & & + & & . & & & + & + & . & + & & & . & . & . &. & 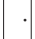 & + & + \\
\hline & Кien.qqa & 10 & & & . & + & & & & & & & & & + & & & & & & & + & . & . & & & . & . & 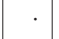 & . & + & + & . \\
\hline & Krenuef & นก & & & . & & & + & & $\cdot$ & & + & . & & & & & & & & & + & . & $\cdot$ & & & . & . & . & $\cdot$ & 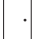 & + & + \\
\hline & $\kappa_{[} \mathrm{n}_{\mathrm{I}}$ & $\stackrel{20}{\circ}$ & & & & & & & &. & & + & & & & & & & & & & & • & . & & & 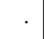 & . & ${ }^{\circ}$ & . & & + & + \\
\hline & ${ }^{\alpha} \mathrm{e} W$ & นొน & & & . & & & & & . & . & + & & & & & & & & & & + & - & - & & & . & . & . & $\cdot$ & & + & + \\
\hline & पग्रू & เి & & & + & & & & + & . & & & . &. & & & & & + & & & & + & . & & & . & $\cdot$ & . & . & & + & $\cdot$ \\
\hline & & 工 & & & . & & + & & & & . & & & & & & + & $\cdot$ & & & 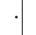 & 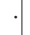 & . & - & & & . & $\cdot$ & . & . & & & . \\
\hline & & $\infty$ & & & . & & & & . & + & . & & & . & . & & & + & & & + & + & + & . & & & . & . & . &. & & & $\cdot$ \\
\hline & $U$ & $\Sigma$ & & + & . & + & & & + & & & & + & + & + & & & + & & + & + & + & + & + & & + & . & . & r. & + & & + & + \\
\hline & & os & & & 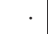 & & & & & 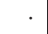 & & & + & & . & 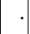 & & + & & & & & 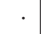 & 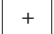 & & & 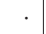 & - & & 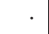 & & & . \\
\hline & 育 & & 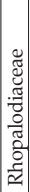 & 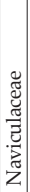 & 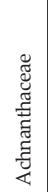 & 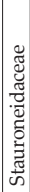 & 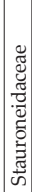 & 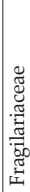 & 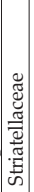 & 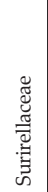 & 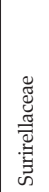 & 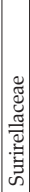 & 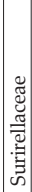 & 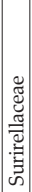 & 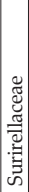 & 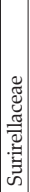 & 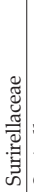 & : & 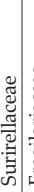 & 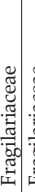 & 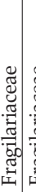 & & 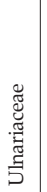 & 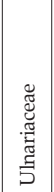 & 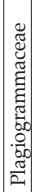 & 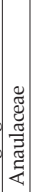 & 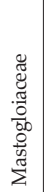 & 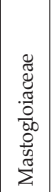 & 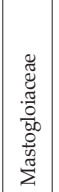 & 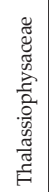 & 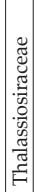 & 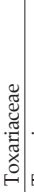 & 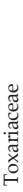 \\
\hline & 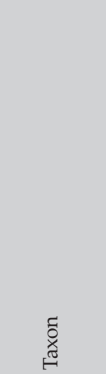 & & 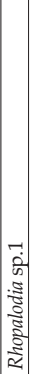 & 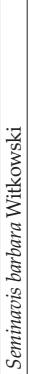 & 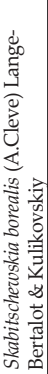 & 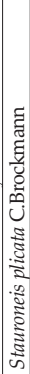 & 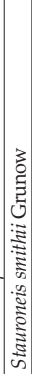 & 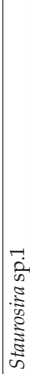 & 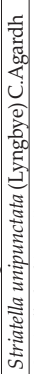 & 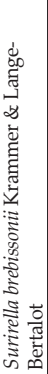 & 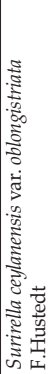 & 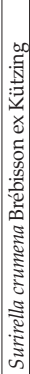 & 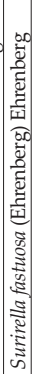 & 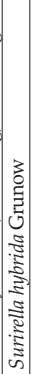 & 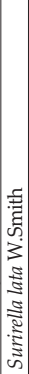 & 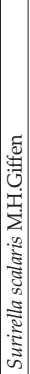 & 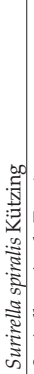 & 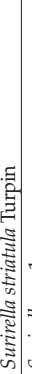 & 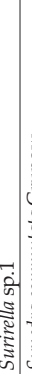 & 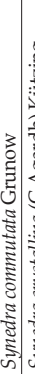 & 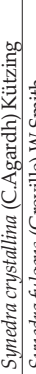 & 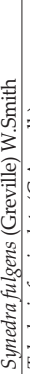 & 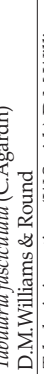 & 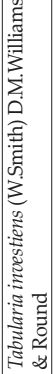 & 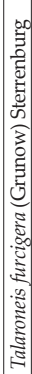 & 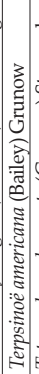 & 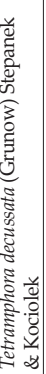 & 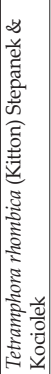 & 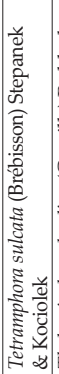 & 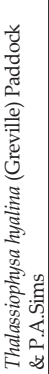 & 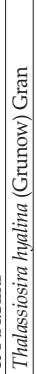 & 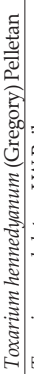 & 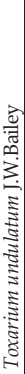 \\
\hline
\end{tabular}




\begin{tabular}{|c|c|c|c|c|c|c|c|c|c|c|c|c|c|c|c|c|c|c|c|}
\hline & (\%) 'bə.⿲丿丨 & & 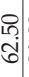 & & & & 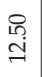 & 周 & $\stackrel{\Delta}{\vec{f}}$ & & $\begin{array}{l}\text { के } \\
\text { ले. }\end{array}$ & & & 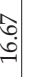 & $\begin{array}{l}\infty \\
\dot{\Delta} \\
\dot{\sim}\end{array}$ & $\begin{array}{c}\infty \\
\infty \\
\infty\end{array}$ & $\underset{+}{\stackrel{s}{+}}$ & $\stackrel{\triangle}{\stackrel{f}{+}}$ & $\begin{array}{l}8 \\
\text { 모 }\end{array}$ \\
\hline & u & & 10 & in & $m$. & + & $m$ & $a$ & - & 9 & $\infty$ & a & $m$. & + & n & N & -1 & - & 6 \\
\hline & & $\infty$ & + & & + & + & $\cdot$ & + & & + & + & $\cdot$ & . & $\cdot$ & $\cdot$ & & $\cdot$ & . &. \\
\hline & मәч & $\stackrel{10}{8}$ & + & & & & $\cdot$ & & & + & & & . & $\cdot$ & . & & $\cdot$ & $\cdot$ &. \\
\hline & & $\infty$ & + & & & & $\cdot$ & $\cdot$ & & & + & $\cdot$ & . & . & $\cdot$ & & $\cdot$ & . & . \\
\hline & 10 q & \begin{tabular}{|c|}
2 \\
\end{tabular} & + & & & &. & + & & & + & & & $\cdot$ & . & &. & $\cdot$ &. \\
\hline & & $\infty$ & + & . & & & $\cdot$ & + & & + & + & . & 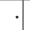 & + & + & & $\cdot$ & . & $\cdot$ \\
\hline & (I) & "ก: & + & & & & $\cdot$ & & & & & & & & $\cdot$ & & $\cdot$ & $\cdot$ & $\cdot$ \\
\hline & 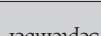 & $\infty$ & + & . & $\cdot$ & 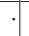 & $\cdot$ & 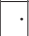 & + & $\cdot 1$ & $\cdot$ & $\cdot$ & $\cdot$ & $\cdot$ & . & & $\cdot$ & $\cdot$ & $\cdot$ \\
\hline & IәquәғаәS & {$\left[\begin{array}{ll}10 \\
0\end{array}\right.$} & + & & & & $\cdot$ & & + & & . & 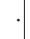 & $\cdot$ & . & . & & $\cdot$ & $\cdot$ & . \\
\hline & & $\infty$ & + & . & & + & $\cdot$ & + & & + & + & $\cdot$ & $\cdot$ & $\cdot$ & $\cdot$ & & $\cdot$ & $\cdot$ & $\cdot$ \\
\hline & 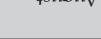 & $\stackrel{10}{0}$ & + & & & & $\cdot$ & + & & & + & $\cdot$ & $\cdot$ & $\cdot$ & . & & $\cdot$ & $\cdot$ & $\cdot$ \\
\hline & & $\infty$ & + & & & . & $\cdot$ & $\cdot$ & & 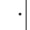 & + & + & · & $\cdot$ & $\cdot$ & . & $\cdot$ & $\cdot$ & . \\
\hline & $x\left[I^{4}\right.$ & เ? & + & . & & & $\cdot$ & + & & & + & & $\cdot$ & $\cdot$ & $\cdot$ & & . & + & $\cdot$ \\
\hline & & $\infty$ & + & + & & + & $\cdot$ & + & & + & + & 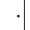 & + & $\cdot$ & $\cdot$ & . & $\cdot$ & . & + \\
\hline & (2) & !n: & + & & & & $\cdot$ & + & & & & & & $\cdot$ & $\cdot$ & & . & $\cdot$ & + \\
\hline & & $\infty$ & 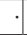 & + & & & + & 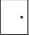 & & & 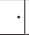 & + & + & + & . & + & . &. &. \\
\hline & Ne $W$ & "? & & + & & & $\cdot$ & & & & & & & $\cdot$ & $\cdot$ & & . & $\cdot$ & $\cdot$ \\
\hline & undur & $\infty$ & 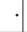 & + & & & $\cdot$ & $\cdot$ & & 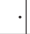 & 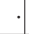 & + & . & + & $\cdot$ & $\cdot$ & . & $\cdot$ & + \\
\hline & & $\stackrel{10}{\circ}$ & & + & & & $\cdot$ & 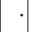 & & & & & + & & . & & . & . & $\cdot$ \\
\hline & чрлеW & เి & & & & & + & + & & t & & & + & . & . & . & . & $\cdot$ & + \\
\hline & KIeniqว & : & & & + & + & $\cdot$ & & + & & & & & . & . & + & . & $\cdot$ & $\cdot$ \\
\hline & Krenue & เి & & & & & $\cdot$ & & & + & & & , & + & . & & . & . & + \\
\hline & ${ }_{\mathrm{S}}^{\mathrm{I}} \mathrm{n} \mathrm{f}$ & : & & & & & . & & & + & & & & & . & & $\cdot$ & $\cdot$ & + \\
\hline$\overline{\mathrm{N}}$ & KeW & $\stackrel{0}{0}$ & + & & & & . & & & + & & & & & . & & + & $\cdot$ & . \\
\hline & पग.еW & : & & & + & & + & & & & & & 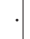 & + & . & & . & $\cdot$ & . \\
\hline & & 工 & & & & & . & & & & & & + & & . & . & + & . & $\cdot$ \\
\hline & & $\infty$ & & & & + & $\cdot$ & & & & + & & + & . & + & + & + & . & . \\
\hline & ज & $\Sigma$ & + & + & + & + & + & + & + & + & + & & + & . & + & + & + & + & + \\
\hline & & os & & & & & $\cdot$ & & & & & & + & . & + & & + & $\cdot$ &. \\
\hline & है & & 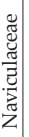 & & . & 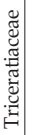 & 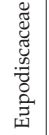 & 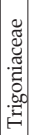 & 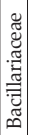 & 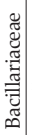 & 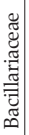 & 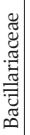 & 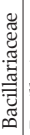 & 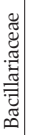 & 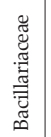 & 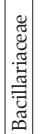 & 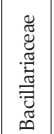 & 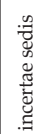 & 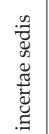 \\
\hline & 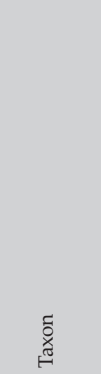 & & 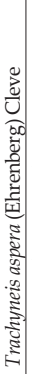 & 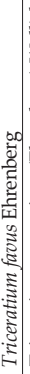 & 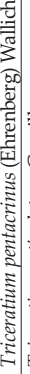 & 党: & 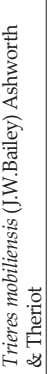 & 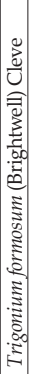 & 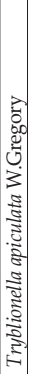 & 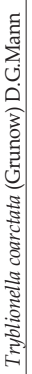 & 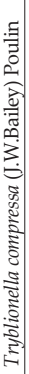 & 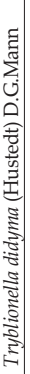 & 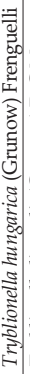 & 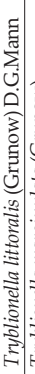 & 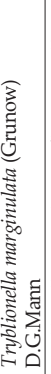 & 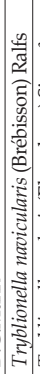 & 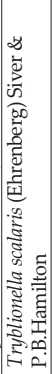 & 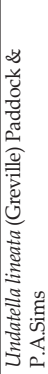 & 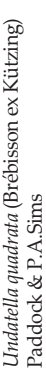 \\
\hline
\end{tabular}




\section{DISCUSSION}

In the present study, small forms belonging to some of the most taxonomically complex genera (e.g. Amphora, Navicula and Nitzschia), characterized by a considerable morphological variability, were recognized (Sullivan \& Currin, 2000). A similar diatom community has been reported for different hard substrates from the Adriatic Basin (Munda, 2005; Tотті et al., 2007) and for coastal rocks of the Mediterranean (cf. ÁlvarezBlanco \& Blanco, 2014). Regarding their ecology, the genus Navicula includes taxa with a very wide ecological range (Krammer \& LANGe-Bertalot, 1991), while Amphora and some Achnanthes taxa are mostly typical of nutrient-poor regions (AgATz et al., 1999). The difficulty in their identification was a major problem in this study. As a matter of fact, more than 40 taxa in Tab. 3 are not fully determined to species level. Additionally, it has been pointed out that some taxa may exhibit more than one growth form, reflecting the different strategies of spatial utilization (cf. RomAGNOLI et al., 2014, and references therein) adopted for the competitive advantage they provide. In this study, although he samples contained a mixture of forms growing on the stones and some epiphytic forms, we refer to this collective community as epilithon (sensu Çolak SABANCI, 2012).

The presence of taxa associated with fresh to brackish waters may be correlated with the precipitation regime and the dynamic water flow from the underground karstic springs ("vruljas") that feed the bay. These taxa were well adapted to this changing environment and these results also support the idea that some species could have different responses to the environmental conditions, resulting in a better or worst adaptation to them (Underwood et al., 1998; Ribeiro et al., 2003; Mino \& WitKowski, 2005).

In our study, the genus Mastogloia was the richest in taxa number, and the most frequent taxa were Amphora bigibba var. interrupta, Cocconeis scutellum, Achnanthes brevipes, Toxarium undulatum and Licmophora remulus. Mastogloia species, one of the largest diatom genera (Pennesi et al., 2011, and references therein), can be found within different biotopes (ÇolaK Sabanci, 2013). In our study, the most frequent Mastogloia was M. cyclops which has been characterized as good indicator of coastal zones (WACHNickA et al., 2010). Some taxa (e.g., C. scutellum, Halamphora coffeiformis) were found on different substrata and do not seem to have a preference either for geographic region or for the substrate type (Romagnoli et al., 2014). By contrast, C. scutellum is considered as typical epiphytic taxon (Ulanova \& SNOEIJS, 2006).

It is difficult to determine the possible seasonal fluctuations in the assemblages based on qualitative data. Many taxa such as Achnanthes brevipes, Actinocyclus subtilis, Grammatophora oceanica and Cocconeis scutellum did not show any kind of marked seasonality. This was also found for several Cocconeis taxa (C. molesta, C. placentula, C. scutellum) in the Venice Lagoon (Tolomio \& Andreoli, 1989). Additionally, ÇolaK Sabanci (2012) in the study based on the seasonal sampling, showed that some of the above mentioned taxa did not have any apparent preference for a given season in the Aegean lagoon in Turkey. In our case, a range of factors can naturally be expected to affect diatom development in the bay, and this research will be investigated in depth in the future.

In our study, the number of taxa was higher than found in floristic surveys conducted in other, similar habitats, in a broad sense and over a wide geographic area in Adriatic or Mediterranean (e.g., Mino \& Wiткоwski, 2005; Çolak Sabanci, 2011, 2012). Other authors obtained quite different results due to differences in the size of the studied areas, environmental conditions and seasons. In addition, special attention must be paid to the use of different cleaning and processing treatments of benthic marine diatom samples 
from Mediterranean oligotrophic areas (VERMEULEN et al., 2012);marine diatoms are likely to be very sensitive to common treatments used in studies of freshwater populations because of the lower availability of silicic acid and of the interference of salinity in silica fixation (CONLEy et al., 1989).

To summarize, this study represents the first floristic list focused on benthic diatoms for Bosnia and Herzegovina. Our analysis shows that, although benthic diatom richness is high in the bay, taxa are apparently not distributed evenly temporally. This is most likely due to the discrete design of the surveys, as many of the rare taxa may occur commonly in unexplored localities of the Adriatic Basin. Also, consistent quantitative and qualitative data are still needed for a better determination of the seasonal and spatial changes of the epilithic assemblages in the region.

From a purely scientific standpoint, it will be intriguing to quantify their contribution to the flow of energy and cycling of material in the bay. More practically, these data will be useful for a rational management of this important regional resource. Namely, the bay is part of a complex ecosystem of the wider area of the Mali Ston Bay (Croatia), which is an important part of the region's natural heritage and they must in fact be protected according to international obligations. Thus, sustainable land management must ensure protection of the centuries-old cultivation of shellfish Ostrea edulis in whole of Mali Ston Bay (VILIČıć, 2017).

\section{AUTHOR CONTRIBUTIONS}

N.J. designed the study and wrote the manuscript, D.H. conducted the field sampling, prepared the samples for counting and analysed the samples using the light microscope, A.C. performed scanning electron microscopy analysis. All authors commented on the manuscript.

\section{ACKNOWLEDGEMENTS}

The authors thank Dr. Rade Garić for drawing Fig. 1, Steve Latham (UK) for improving the English, and two anonymous reviewers whose observations improved the quality of the final version of the manuscript. This research was supported by Croatian Ministry of Science, Education and Sports (project 275-0000000-3186) and by Croatian science foundation (HRZZ, IP-2014-09-2945).

Received August 6, 2017

\section{REFERENCES}

Agatz, M., Asmus, R.M \& Deventer, B., 1999: Structural changes in the benthic diatom community along a eutrophication gradient on a tidal flat. Helgoland Marine Research 53, 92-101.

Álvarez-Blanco, I. \& Blanco, S., 2014: Benthic diatoms from Mediterranean coasts. Bibliotheca Diatomologica 60, 1-409.

Bartole, L., Welker, C., Dellavalle, G. \& Bressan, G., 1991-1994: Primi stadi di colonizzazione “a diatomee" di un substrato duro artificiale in due stazioni fisse del Golfo di Trieste (nord Adriatico). Nova Thalassia 12, 163-189.

Bérard-Therriault, L., Cardinal, A. \& Poulin, M., 1986: Les diatomées (Bacillariophyceae) benthiques de substrats durs des eaux marines et saumâtres du Québec. 6. Naviculales: Cymbellaceae et Gomphonemaceae. Naturaliste Canadien (Revue d'Ecologie et de Systématique) 113, 405-429. 
Bérard-Therriault, L., Cardinal, A. \& Poulin, M., 1987: Les diatomées (Bacillariophyceae) benthiques de substrats durs des eaux marines et saumâtres du Québec. 8. Centrales. Naturaliste Canadien (Revue d'Ecologie et de Systématique) 114, 81-113.

Blake, G.H. \& Topalović, D., 1996: Maritime boundaries on the Adriatic Sea. Maritime Briefing, Volume 1, No 8.

Burić, Z., CAPUT, K. \& ViličIĆ, D., 2004: Distribution of the diatom Cocconeis scutellum in the karstic estuary (Zrmanja, eastern Adriatic Sea). Biologia 59, 1-7.

Caput Mihalić, K., Viličić, D., Ahel, M., Burić, Z. \& Carić, M., 2008: Periphytic algae development in the upper reach of the Zrmanja Estuary (eastern Adriatic coast). Vie Milieu 58, 203-213.

Car, A., Witkowski, A., Dobosz, S., Burfeind, D.D., Meinesz, A., Jasprica, N., Ruppel, M., KurzydŁowski, J.K. \& PŁocıński, T., 2012: Description of a new marine diatom Cocconeis caulerpacola sp. nov. (Bacillariophyceae), epiphytic on invasive Caulerpa species. European Journal of Phycology 47, 433-448.

Cibic, T. \& FAccA, C., 2010: Microphytobenthos. In: Relini, G. (ed.), Checklist della flora e della fauna dei mari italiani. Parte II. Biologia Marina Mediterranea 17 (suppl. 1), 754-800.

Cibic, T. \& Blasutto, O., 2011: Living marine benthic diatoms as indicators of nutrient enrichment: a case study in the Gulf of Trieste. In: Compton, J. C. (ed.), Diatoms: Ecology and Life Cycle. Hauppauge, New York: Nova Science Publishers, pp. 169-184.

Çolak SabancI, F., 2011: Relationship of epilithic diatom communities to environmental variables in Homa lagoon (Izmir, Turkey). Aquatic Biology 13: 233-241.

ÇolAK SABANCI, F., 2012: Taxonomic survey of benthic diatoms on natural substrata from coastal lagoon (Aegean Sea, Turkey). Turkish Journal of Fisheries and Aquatic Sciences 12, 841-849.

ÇOlAK SABANCI, F., 2013: Species of Mastogloia (Bacillariophyceae) - new for the Aegean coast of Turkey. Mediterranean Marine Science 14, 129-140.

Çolak Sabanci, F. \& Koray, T., 2010: Four new records for the benthic diatoms (genera Cocconeis, Seminavis, Synedra and Trachysphenia) from the Aegean Sea. Turkish Journal of Botany 34, 531-540.

Conley, D.J., Kilham, S.S. \& Theriot, E., 1989: Differences in silica content between marine and freshwater diatoms. Limnology and Oceanography 34, 205-213.

Čalić, M., Carić, M., Kršinić, F., Jasprica, N. \& Pećarević, M., 2013: Controlling factors of phytoplankton seasonal succession in oligotrophic Mali Ston Bay (south-eastern Adriatic). Environmental Monitoring and Assessment 185, 7543-7563.

Dupčić Radić, I., Hrustić, E. \& Jasprica, N., 2013: Nutrient and chlorophyll a concentrations in the Neum Bay (Bosnia and Herzegovina). In: Alegro, A. \& Boršıć, I. (eds.), Abstracts of the $4^{\text {th }}$ Croatian Botanical Symposium with international participation, Split 2013. Split: Croatian Botanical Society, pp. 55.

Facca, C., Sfriso, A. \& Socal, G., 2002: Temporal and spatial distribution of diatoms in the surface sediments of the Venice Lagoon. Botanica Marina 45, 170-183.

FACCA, C. \& SFriso, A., 2007: Epipelic diatom spatial and temporal distribution and relationship with the main environmental parameters in coastal waters. Estuarine, Coastal and Shelf Science 75, 35-49.

Falkowski, P.G., Schofield, O., Katz, M.E., Van de Schootbrugge, B. \& Knoll, A.H., 2004: Why is the land green and the ocean red? In: Thierstein, H.R. \& Young, J.R. (eds.), Coccolithophores. From molecular processes to global impacy. Berlin: Springer, pp. 429-453.

Franzo, A., Cibic, T., Del Negro, P. \& De Vittor, C., 2015: Spatial distribution of microphytobenthos, meiofauna and macrofauna in the north-western Adriatic Sea: a synoptic study. Advances in Oceanography and Limnology 6, 58-75.

Grasshoff, K., Ehrhardt, M. \& Kremling, K., 1983: Methods of seawater analysis. 2nd editon. Weinheim: Verlag Chemie GmbH.

Guiry, M.D. \& GuIRY, G.M., 2017: AlgaeBase. World-wide electronic publication, National University of Ireland, Galway. http://www.algaebase.org [Last access: 20 October 2017].

Hartley, B., 1986: A check-list of the freshwater, brackish and marine diatoms of the British Isles and adjoining coastal waters. Journal of the Marine Biological Association of the United Kingdom 66, 531-610.

Hartley, B., Barber, H.G. \& Carter, J.R., 1996: An Atlas of British Diatoms. Bristol: Biopress.

Hasle, G.R. \& Fryxell, G.A., 1970: Diatoms: cleaning and mounting for light and electron microscopy. Transactions of the American Microscopical Society 84, 469-474.

Hendey, N.I., 1964: An introductory account of the smaller algae of British coastal waters. Part V. Bacillariophyceae (Diatoms). Ministry of Agriculture, Fisheries and Food, Fisheries Investigations, Seies IV, London, 317 pp. 
JASPRICA, N., 1989: Distribution of phytoplankton population density and volume-biomass in the Mali Ston and Gruž Bays (the southern Adriatic). Ekologija 24, 83-96.

Jasprica, N., Carić, M, Bolotin, J. \& Rudenjak-Lukenda, M., 1997: The Mediterranean mussel (Mytilus galloprovincialis Lmk.) growth rate response to phytoplankton and microzooplankton densities in the Mali Ston Bay (Southern Adriatic). Periodicum biologorum 99, 255-264.

JaspricA, N. \& CARIĆ, M., 2001: Planktonic diatoms and their relation to environmental factors at three stations in the Southern Adriatic, Mediterranean Sea. In: JAhn, R., J. P. Koсiolek, A. Witкowski \& P. Compère (eds.), Lange-Bertalot-Festschrift: Studies on Diatoms, Ruggell: Gantner, pp. 513-536.

Jasprica, N., Carić, M., Kršinić, F., Kapetanović, T., Batistić, M. \& NJire, J., 2012: Planktonic diatoms and their environment in the lower Neretva River estuary (Eastern Adriatic Sea, NE Mediterranean). Nova Hedwigia, Beiheft. 141, 405-430.

Krammer, K. \& Lange-Bertalot, H., 1991: Bacillariophyceae: Achnanthaceae, Kritische Ergänzungen zu Navicula (Lineolatae) und Gomphonema. Gesamtliteraturverzeichnis. In: Ettr, H., Gerloff, J., Heynig, H. \& Mollenhauer, D. (eds.), Süßwasserflora von Mitteleuropa, Vol. 2/4. Stuttgart: Gustav Fischer Verlag, pp. 1-437.

Levkov, Z., Caput Minalić, K. \& Ector, L., 2010: A taxonomical study of Rhoicosphenia Grunow (Bacillariophyceae) with a key for identification of selected taxa. Fottea 10, 145-200.

Medlin, L.K. \& Kaczmarska, I., 2004: Evolution of the diatoms: V. Morphological and cytological support for the major clades and a taxonomic revision. Phycologia 43, 245-270.

Mejdandžić, M., Ivanković, T., Pfannkuchen, M., Godrijan, J., Marić Pfannkuchen, D., Hrenović, J. \& LJubešıć, Z., 2015: Colonization of diatoms and bacteria on artificial substrates in the northeastern coastal Adriatic Sea. Acta Botanica Croatica 74, 407-422.

Miно, A. \& Wiткоwsкi, A., 2005: Diatom (Bacillariophyta) flora of Albanian coastal wetlands taxonomy and ecology: A review. Proceedings of the California Academy of Sciences 56, 129-145.

MundA, I.M., 2005: Seasonal fouling by diatoms on artificial substrata at different depths near Piran (Gulf of Trieste, Northern Adriatic). Acta Adriatica 46, 137-157.

Nenadović, T., Šarčević, T., Čižmek, H., Godrijan, J., Marić Pfannkuchen, D., Pfannkuchen, M. \& Ljubešıć, Z., 2015: Development of periphytic diatoms on different artificial substrates in the Eastern Adriatic Sea. Acta Botanica Croatica 74, 377-392.

Pennesi, C., Poulin, M., De Stefano, M., Romagnoli, T. \& Totti, C., 2011: New insights to the ultrastructure of some marine Mastogloia species section Sulcatae (Bacillariophyceae), including Mastogloia neoborneensis sp. nov. Phycologia 50, 548-562.

Peragallo, H. \& Peragallo, M., 1897-1908: Diatomées marines de France et des districts maritimes voisin. Micrographe-Editeur, Grez-sur-Loing (S.-et-M.), 491 pp.

Pikelj, K. \& Juračić, M., 2013: Eastern Adriatic coast (EAC): Geomorphology and coastal vulnerability of a karstic coast. Journal of Coastal Research 29, 944-957.

Poulin, M., Bérard-Therriault, L. \& Cardinal, A., 1984: Les diatomées benthiques de substrats durs des eaux marines et saumâtres du Québec. 3. Fragilarioideae (Fragilariales, Fragilariaceae). Naturaliste Canadien (Revue d'Ecologie et de Systématique) 111, 349-367.

Poulin, M., Bérard-Therriault, L., Cardinal, A. \& Hamilton, P.B., 1990: Les diatomées (Bacillariophyta) benthiques de substrats durs des eaux marines et saumâtres du Québec. 9. Bacillariaceae. Naturaliste Canadien (Revue d'Ecologie et de Systématique) 117, 73-101.

Ribeiro, L., Brotas, V., Mascarell, G. \& Couté, A., 2003: Taxonomic survey of the microphytobenthic communities of two Tagus estuary mudflats. Acta Oecologica 24, 117-123.

RicARD, M., 1974: Etude taxonomique des diatomées marines du lagon de Vairao (Tahiti) 1. Le genre Mastogloia. Revue Algologique, nouvelle série 11, 161-177.

RicArD, M., 1975: Quelques diatomées nouvelles de Tahiti décrites en microscopie photonique et électronique à balayage. Bulletin du Musée National d'Histoire Naturelle, 3e serie, 326, 201- 229.

RicArd, M., 1977: Les peuplements de diatomeés des lagons de l'Archipel de la Société (Polynésie Française). Revue Algologique, nouvelle série 12, 137-336.

Romagnoli, T., Bavestrello, G., Cucchiari, E.M., De Stefano, M., Di Camillo, C., Pennesi, C., Puce, S. \& Tотті, C., 2007: Microalgal communities epibiontic on the marine hydroid Eudendrium racemosum in the Ligurian Sea during an annual cycle. Marine Biology 151, 537- 552.

Romagnoli, T., Totti, C., Accoroni, S., De Stefano, M. \& Pennesi, C., 2014: SEM analysis of the epibenthic diatoms on Eudendrium racemosum (Hydrozoa) from the Mediterranean Sea. Turkish Journal of Botany 38, 566-594. 
Sdrigotti, E., Barbariol, V. \& Welker, C., 1999: Diatom assemblages in coastal shallow waters at the water-sediment interface (Gulf of Trieste, north Adriatic Sea). Annals for Istrian and Mediterranean Studies, Series historia naturalis 2, 191-202.

SNoeIJs, P., 1993: Intercalibration and distribution of diatoms in the Baltic Sea, 1, 1-129. Baltic Marine Biologists Publication 16a, Uppsala: Opulus Press.

Snoeijs, P., 1999: Marine and brackish waters. In: Snoeijs, P. \& M. Diekmann (eds.), Swedish Plant Geography. Acta Phytogeogr. Suec. 84. Uppsala: Opulus Press. pp. 187-212.

Snoeijs, P. \& Balashlova, N., 1998: Intercalibration and distribution of diatoms in the Baltic Sea, 5, 1-144. Baltic Marine Biologists Publication 16e, Uppsala: Opulus Press.

Snoeijs, P. \& Kasperoviciené, J., 1996: Intercalibration and distribution of diatoms in the Baltic Sea, 4, 1-125. Baltic Marine Biologists Publication 16d, Uppsala: Opulus Press.

Snoeijs, P. \& Potapova, M., 1995: Intercalibration and distribution of diatoms in the Baltic Sea, 3, 1-125. Baltic Marine Biologists Publication 16c, Uppsala: Opulus Press.

Stevenson, R. J. \& PAN, Y., 1999: Assessing environmental conditions in rivers and streams with diatoms. In: Stoermer E. F. \& J. P. Smol (eds.), The Diatoms. Application for the environmental and earth sciences. Cambridge: University Press, pp. 469.

Sullivan, M.J. \& Currin, C.A., 2000. Community structure and functional dynamics of benthic microalgae in salt marshes. In: Weinstein M. P. \& Kreeger, D. A. (eds.), Concepts and controversies in tidal marsh ecology. Dordrecht: Kluwer Academic Publishers, pp. 81-106.

Tolomio, C. \& Andreoli, C., 1989: Recherches sur le periphyton à diatomées dans un vivier de la Lagune de Venise (mai 1984-mai 1985). Diatom Research 4, 151-162.

Tolomio, C., Moro, I., Moschin, E. \& Valandro, A., 1999: Résultats préliminaires sur les diatomées benthiques de substrats meubles dans la lagune de Venise, Italie (Mars 1994-Janvier 1995). Diatom Research 14, 367-379.

Tolomio, C., Moschin, E. \& Duzzin, B., 2002: Distribution des diatomées benthiques de substrats meubles dans le bassin sud de la lagune de Venise, Italie. Diatom Research 17, 401-414.

Тотті, C., 2003: Influence of the plume of the river Po on the distribution of subtidal microphytobenthos in the northern Adriatic Sea. Botanica Marina 46, 161-178.

Totti, C., Cucchiari, E., De Stefano, M., Pennesi, C., Romagnoli, T. \& Bavestrello, G., 2007: Seasonal variations of epilithic diatoms on different hard substrates, in the northern Adriatic Sea. Journal of the Marine Biological Association of the United Kingdom 87, 649-658.

Ulanova, A. \& Snoeijs, P., 2006: Gradient responses of epilithic diatom communities in the Baltic Sea proper. Estuarine, Coastal and Shelf Science 68, 661-674.

Underwood, G.J.C., Phillips, J. \& Saunders, K., 1998: Distribution of estuarine benthic diatom species along salinity and nutrient gradients. European Journal of Phycology 33, 173-183.

Vermeulen, S., Lepoint, G. \& Gobert, S., 2012: Processing samples of benthic marine diatoms from Mediterranean oligotrophic areas. Journal of Applied Phycology 24, 1253-1260.

Viličıć, D., 2017: Protection of oyster farming region from anthropogenic influence in the Mali Ston Bay (SE Adriatic coast). Hrvatske vode 25, 59-64 (in Croatian).

Wachnicka, A., Gaiser, E., Collins, L., Frankovich, T. \& Boyer, J., 2010: Distribution of diatoms and development of diatom-based models for inferring salinity and nutrient concentrations in Florida Bay and adjacent coastal wetlands of South Florida (USA). Estuaries and Coasts 33, 1080-1098.

Winter, J.G. \& Duthie, H.C., 2000: Stream epilithic, epipelic and epiphytic diatoms: habitat fidelity and use in biomonitoring. Aquatic Ecology 34, 345-353.

Witkowski, A., Lange-Bertalot, H. \& Metzelin, D., 2000: Diatom flora of marine coasts I. In: Iconographia diatomologica, vol. 7, Lange-Bertalot, H. (ed.), Ruggell, Liechtenstein: A.R.G. Gantner. 